\title{
Efficiency of radial transport of ices in protoplanetary disks probed with infrared observations: the case of $\mathrm{CO}_{2}$
}

\author{
Arthur D. Bosman ${ }^{1}$, Alexander G. G. M. Tielens ${ }^{1}$, and Ewine F. van Dishoeck ${ }^{1,2}$ \\ ${ }^{1}$ Leiden Observatory, Leiden University, PO Box 9513, 2300 RA Leiden, The Netherlands \\ e-mail: bosman@strw. leidenuniv.nl \\ ${ }^{2}$ Max-Planck-Insitut für Extraterrestrische Physik, Gießenbachstrasse 1, 85748 Garching, Germany
}

Received 6 October 2017 / Accepted 7 December 2017

\begin{abstract}
Context. Radial transport of icy solid material from the cold outer disk to the warm inner disk is thought to be important for planet formation. However, the efficiency at which this happens is currently unconstrained. Efficient radial transport of icy dust grains could significantly alter the composition of the gas in the inner disk, enhancing the gas-phase abundances of the major ice constituents such as $\mathrm{H}_{2} \mathrm{O}$ and $\mathrm{CO}_{2}$.

Aims. Our aim is to model the gaseous $\mathrm{CO}_{2}$ abundance in the inner disk and use this to probe the efficiency of icy dust transport in a viscous disk. From the model predictions, infrared $\mathrm{CO}_{2}$ spectra are simulated and features that could be tracers of icy $\mathrm{CO}_{2}$, and thus dust, radial transport efficiency are investigated.

Methods. We have developed a 1D viscous disk model that includes gas accretion and gas diffusion as well as a description for grain growth and grain transport. Sublimation and freeze-out of $\mathrm{CO}_{2}$ and $\mathrm{H}_{2} \mathrm{O}$ has been included as well as a parametrisation of the $\mathrm{CO}_{2}$ chemistry. The thermo-chemical code DALI was used to model the mid-infrared spectrum of $\mathrm{CO}_{2}$, as can be observed with JWSTMIRI.

Results. $\mathrm{CO}_{2}$ ice sublimating at the iceline increases the gaseous $\mathrm{CO}_{2}$ abundance to levels equal to the $\mathrm{CO}_{2}$ ice abundance of $\sim 10^{-5}$, which is three orders of magnitude more than the gaseous $\mathrm{CO}_{2}$ abundances of $\sim 10^{-8}$ observed by Spitzer. Grain growth and radial drift increase the rate at which $\mathrm{CO}_{2}$ is transported over the iceline and thus the gaseous $\mathrm{CO}_{2}$ abundance, further exacerbating the problem. In the case without radial drift, a $\mathrm{CO}_{2}$ destruction rate of at least $10^{-11} \mathrm{~s}^{-1}$ or a destruction timescale of at most $1000 \mathrm{yr}$ is needed to reconcile model prediction with observations. This rate is at least two orders of magnitude higher than the fastest destruction rate included in chemical databases. A range of potential physical mechanisms to explain the low observed $\mathrm{CO}_{2}$ abundances are discussed. Conclusions. We conclude that transport processes in disks can have profound effects on the abundances of species in the inner disk such as $\mathrm{CO}_{2}$. The discrepancy between our model and observations either suggests frequent shocks in the inner $10 \mathrm{AU}$ that destroy $\mathrm{CO}_{2}$, or that the abundant midplane $\mathrm{CO}_{2}$ is hidden from our view by an optically thick column of low abundance $\mathrm{CO}_{2}$ due to strong $\mathrm{UV}$ and/or X-rays in the surface layers. Modelling and observations of other molecules, such as $\mathrm{CH}_{4}$ or $\mathrm{NH}_{3}$, can give further handles on the rate of mass transport.
\end{abstract}

Key words. astrochemistry - accretion, accretion disks - methods: numerical - protoplanetary disks

\section{Introduction}

To date, a few thousand planetary systems have been found ${ }^{1}$. Most of them have system architectures that are very different from our own solar system (Madhusudhan et al. 2014) and explaining the large variety of systems is a challenge for current planet formation theories (see, e.g. Morbidelli \& Raymond 2016, and references therein). Thus, the birth environment of planets protoplanetary disks - are an active area of study. A young stellar system inherits small dust grains from the interstellar medium. In regions with high densities and low turbulence, the grains start to coagulate. In the midplane of protoplanetary disks, where densities are higher than $10^{8} \mathrm{~cm}^{-3}$, grain growth can really take off. Grain growth and the interactions of these grown particles with the gaseous disk are of special interest to planet formation (see, e.g. Weidenschilling 1977; Lambrechts \& Johansen 2012; Testi et al. 2014). The growth of dust grains to comets and planets is far from straightforward, however.

exoplanets. org as of 28 November 2017.
Pebbles, that is, particles that are large enough to slightly decouple from the gas, have been invoked to assist the formation of planets in different ways (Johansen \& Lambrechts 2017). They are not supported by the pressure gradient from the gas, but they are subject to gas drag. As a result pebbles drift on a timescale that is an order of magnitude faster than the gas depletion time-scale. This flow of pebbles, if intercepted or stopped, can help with planet formation. The accretion of pebbles onto forming giant planetary cores should help these cores grow beyond their classical isolation mass (Ormel \& Klahr 2010; Lambrechts \& Johansen 2012) while the interactions of gas and pebbles near the inner edge of the disk can help with the formation of ultra compact planetary systems as found by the Kepler satellite (Tan et al. 2016; Ormel et al. 2017). Efficient creation and redistribution of pebbles would lead to quick depletion of the solid content of disks increasing their gas-to-dust ratios by one to two orders of magnitude in $1 \mathrm{Myr}$ (e.g. Ciesla \& Cuzzi 2006; Brauer et al. 2008; Birnstiel et al. 2010).

Observations of disks do not show evidence of strong dust depletion. The three disks that have far-infrared measurements of the HD molecule to constrain the gas content show gas-to-dust 
ratios smaller than 200 (Bergin et al. 2013; McClure et al. 2016; Trapman et al. 2017), including the $10 \mathrm{Myr}$ old disk TW Hya (Debes et al. 2013). Gas-to-dust ratios found from gas mass estimates using CO line fluxes are inconsistent with strong dust depletion as well (e.g. Ansdell et al. 2016; Miotello et al. 2017). Manara et al. (2016) also argue from the observed relation between accretion rates and dust masses, that the gas-to-dust ratio in the 2-3 Myr old Lupus region should be close to 100 .

It is thus of paramount importance that a way is found to quantify the inwards mass flux of solid material due to radial drift from observations. This is especially important for the inner $(<10 \mathrm{AU})$ regions of protoplanetary disks. Here we propose to look for a signature of efficient radial drift in protoplanetary disks through molecules that are a major component of icy planetesimals.

Radial drift is expected to transport large amounts of ice over the iceline, depositing a certain species in the gas phase just inside and ice just outside the iceline (Stevenson \& Lunine 1988; Piso et al. 2015; Öberg \& Bergin 2016). This has been modelled in detail for the water iceline by Ciesla \& Cuzzi (2006) and Schoonenberg \& Ormel (2017), for the CO iceline by Stammler et al. (2017) and in general for the $\mathrm{H}_{2} \mathrm{O}, \mathrm{CO}$ and $\mathrm{CO}_{2}$ icelines by Booth et al. (2017). In all cases the gaseous abundance of a molecule in the ice is enhanced inside of the iceline as long as there is an influx of drifting particles. The absolute value and width of the enhancement depend on the mass influx of ice and the strength of viscous mixing. Such an enhancement may be seen directly with observations of molecular lines. Out of the three most abundant ice species $\left(\mathrm{CO}, \mathrm{H}_{2} \mathrm{O}\right.$ and $\left.\mathrm{CO}_{2}\right), \mathrm{CO}_{2}$ is the most promising candidate for a study of this nature. Both $\mathrm{CO}$ and $\mathrm{H}_{2} \mathrm{O}$ are expected to be highly abundant in the inner disk based on chemical models (see, e.g. Aikawa \& Herbst 1999; Markwick et al. 2002; Walsh et al. 2015; Eistrup et al. 2016). As such any effect of radial transport of icy material will be difficult to observe. $\mathrm{CO}_{2}$ is expected to be abundant in outer disk ices (with abundances around $10^{-5}$ if inherited from the cloud or produced in situ in the disk, Pontoppidan et al. 2008; Boogert et al. 2015; Le Roy et al. 2015; Drozdovskaya et al. 2016), but it is far less abundant in the gas in the inner regions of the disk (with abundances around $10^{-8}$, Pontoppidan et al. 2010; Bosman et al. 2017). This gives a leverage of three orders of magnitude to see effects from drifting icy pebbles.

Models by Cyr et al. (1998) and Ali-Dib et al. (2014), for example, predict depletion of volatiles in the inner disk. In these models, all volatiles are locked up outside of the iceline in stationary solids. In Ali-Dib et al. (2014) this effect is strengthened by the assumption that the gas and the small dust in the disk midplane are moving radially outwards such as proposed by Takeuchi \& Lin (2002). Together this leads to very low inner disk $\mathrm{H}_{2} \mathrm{O}$ abundances in their models. $\mathrm{CO}_{2}$ is not included in these models, but the process for $\mathrm{H}_{2} \mathrm{O}$ would also work for $\mathrm{CO}_{2}$, but slightly slower, as the $\mathrm{CO}_{2}$ iceline is slightly further out. However, these models do not include the diffusion of small grains through the disk, which could resupply the inner disk with volatiles at a higher rate than that caused by the radial drift of large grains.

Bosman et al. (2017) showed that an enhancement of $\mathrm{CO}_{2}$ near the iceline would be observable in the ${ }^{13} \mathrm{CO}_{2}$ mid infrared spectrum, if that material were mixed up to the upper layer. Here we continue on this line of research by constraining the maximal mass transport rate across the iceline and by investigating the shape and amplitude of a possible $\mathrm{CO}_{2}$ abundance enhancement near the iceline due to this mass transport.
To constrain the mass transport we have build a model along the same lines as Ciesla \& Cuzzi (2006) and Booth et al. (2017) except that we do not include planet formation processes. As in Booth et al. (2017) we use the dust evolution prescription from Birnstiel et al. (2012). The focus is on the chemistry within the $\mathrm{CO}_{2}$ iceline to make predictions on the $\mathrm{CO}_{2}$ content of the inner disk. In contrast with Schoonenberg \& Ormel (2017) a global disk model is used to maintain consistency between the transported mass and observed outer disk masses. Chemical studies of the gas in the inner disk have been presented by, for example Agúndez et al. (2008), Eistrup et al. (2016), and Walsh et al. (2015) but transport processes are not included in these studies. Cridland et al. (2017) use an evolving disk, including grain growth and transport, coupled with a full chemical model in their planet formation models. However, they do not include transport of ice and gas species due to the various physical evolution processes.

Section 2 presents the details of our physical model, whereas Sect. 3 discusses various midplane chemical processes involving $\mathrm{CO}_{2}$ and our method for simulating infrared spectra. Section 4 presents the model results for a range of model assumptions and parameters. One common outcome of all of these models is that the $\mathrm{CO}_{2}$ abundance in the inner disk is very high, orders of magnitude more than observed, making it difficult to quantify mass transport. Section 5 discusses possible ways to mitigate this discrepancy and the implications for the physical and chemical structure of disks, suggesting JWST-MIRI observations of ${ }^{13} \mathrm{CO}_{2}$ that can test them.

\section{Physical model}

\subsection{Gas dynamics}

To investigate the effect of drifting pebbles on inner disk gasphase abundances we build a 1D dynamic model. This model starts with an $\alpha$-disk model (Shakura \& Sunyaev 1973). The evolution of the surface density of gas $\Sigma_{\text {gas }}(t, r)$ can be described as:

$\frac{\partial \Sigma_{\mathrm{gas}}}{\partial t}=\frac{3}{r} \frac{\partial}{\partial r}\left[r^{1 / 2} \frac{\partial}{\partial r}\left(\frac{\alpha c_{s}^{2}}{\Omega} \Sigma_{\mathrm{gas}} r^{1 / 2}\right)\right]$,

where $r$ is the distance to the star, $t$ is time, $\alpha$ is the dimen-

sionless Sakura-Sunyaev parameter, $\Omega$ is the local Keplerian frequency and $c_{s}=\sqrt{k_{b} T / \mu}$ is the local sound speed, with $k_{b}$ the Boltzmann constant, $\mathrm{T}$ the local temperature and $\mu$ the mean molecular mass which is taken to be $2.6 \mathrm{amu} . \alpha c_{s}^{2} / \Omega$ is also equal to $v_{\text {turb }}$, the (turbulent) gas viscosity. The viscosity, or the resistance of the gas to shear, is responsible for the exchange of angular momentum. The origin of the viscosity is generally assumed to be turbulence stirred up by the magneto-rotational instability (MRI; Turner et al. 2014) although Eq. (1) is in principle agnostic to the origin of the viscosity as long as the correct $\alpha$ value is included. For the rest of the paper, we assume that the viscosity originates from turbulence and that the process responsible for the viscous evolution is also the dominant process in mixing constituents of the disk radially.

The evolution of the surface density of a trace quantity has two main components. First, all gaseous constituents are moving with the flow of the gas. This advection is governed by:

$$
\frac{\partial \Sigma_{x, \mathrm{gas}}}{\partial t}=\frac{1}{r} \frac{\partial}{\partial r}\left(F_{\mathrm{gas}} r \frac{\Sigma_{x, \mathrm{gas}}}{\Sigma_{\mathrm{gas}}}\right),
$$


where $F_{\text {gas }}$ is the total radial flux, which is related to the radial velocity of the gas due to viscous accretion, $u_{\text {gas }}$, and is given by:

$F_{\text {gas }}=\Sigma_{\text {gas }} u_{\text {gas }}=\frac{3}{\sqrt{r}} \frac{\partial}{\partial r} v \Sigma_{\text {gas }} \sqrt{r}$.

Second, the gas is also mixed by the turbulence, smoothing out variations in abundance. This diffusion can be written as (Clarke \& Pringle 1988; Desch et al. 2017)

$\frac{\partial \Sigma_{x, \mathrm{gas}}}{\partial t}=\frac{1}{r} \frac{\partial}{\partial r}\left(r D_{x} \Sigma_{\mathrm{gas}} \frac{\partial}{\partial r}\left(\frac{\Sigma_{x, \mathrm{gas}}}{\Sigma_{\mathrm{gas}}}\right)\right)$,

where $D_{x}$ is the gas mass diffusion coefficient. The diffusivity is related to the viscosity by:

$\mathrm{Sc}=\frac{v_{\mathrm{gas}}}{D_{x}}$

with Sc the Schmidt number. For turbulent diffusion in a viscous disk it is expected to be of order unity. As such, Sc $=1$ is assumed for all gaseous components.

Advection and diffusion are both effective in changing the abundance of a trace species if an abundance gradient exists. Diffusion is most effective in the presence of strong abundance gradients and strong changes in the abundance gradient, such as near the iceline. At the icelines the diffusion of a trace species will generally dominate over the advection due to gas flow.

\subsection{Dust growth and dynamics}

The dynamics of dust grains are strongly dependent on the grain size. A grain with a large surface area relative to its mass is well coupled to the gas and will not act significantly different from a molecule in the gas. Solid bodies with a very small surface area relative to its mass, for example, planetesimals, will not be influenced by the gas pressure or turbulence, their motions are then completely governed by gravitational interactions. Dust particles with sizes between these extremes will be influenced by the presence of the gas in various ways. To quantify these regimes it is useful to consider a quantity known as the Stokes number: The Stokes number is, assuming Epstein drag and spherical particles in a vertically hydrostatic disk, given by (Weidenschilling 1977; Birnstiel et al. 2010):

$\mathrm{St}=\frac{a_{\mathrm{grain}} \rho_{s}}{\Sigma_{\mathrm{gas}}} \frac{\pi}{2}$.

Particles with a very small Stokes number $(\ll 1)$ are very well coupled to the gas and the gas pressure gradients and particles with very large Stokes number $(\gg 1)$ are decoupled from the gas.

The coupling between gas and dust determines both the diffusivity of the dust, that is, how well the dust mixed due to turbulent motions of the disk as well as advection of the dust due to bulk flows of the gas. Youdin \& Lithwick (2007) derived that the diffusivity $D_{\text {dust }}$ of a particle with a certain Stokes number can be related to the gas diffusivity by:

$D_{\text {dust }}=\frac{D_{\text {gas }}}{1+\mathrm{St}^{2}}$

Similarly the advection speed of dust due to gas advection can be given by:

$u_{\text {dust,adv }}=\frac{u_{\text {gas }}}{1+\mathrm{St}^{2}}$.
When particles are not completely coupled to the gas they are also no longer completely supported by the gas pressure gradient. The gas pressure gradient, from high temperature, high density material close to the star, to low temperature, low density material far away from the star provides an outwards force, such that the velocity with which the gas has a stable orbit around the star is lower than the Keplerian velocity. Particles that start to decouple from the gas thus also need to speed up relative to the gas to stay in a circular orbit. This induces a velocity difference between the gas and the dust particles. The velocity difference between the gas velocity and a Keplerian orbital velocity is given by:

$\Delta u=\Omega r-\sqrt{\Omega^{2} r^{2}-\frac{r}{\rho_{\mathrm{gas}}} \frac{\partial P}{\partial r}}$,

with $P$ the pressure of the gas. We note that in the case of a positive pressure gradient, the gas will be moving faster than the Keplerian velocity

As a result of this velocity difference the particles are subjected to a drag force, which, in the case of a negative pressure gradient, removes angular momentum from the particles. The loss of angular momentum results in an inwards spiral of the dust particles. This process is known as radial drift. The maximal drift velocity can be given as (Weidenschilling 1977):

$u_{\mathrm{drift}}=\frac{2 \Delta u}{\mathrm{St}+\mathrm{St}^{-1}}$.

The drift velocity will thus be maximal for particles with a Stokes number of unity. Drift velocities of $\sim 1 \%$ of the orbital velocity are typical for particles with a Stokes number of unity.

The dynamics of dust are thus intrinsically linked to the size, or rather size distribution of the dust particles. The dust size distribution is set by the competition of coagulation and fragmentation $^{2}$. When two particles collide in the gas they can either coagulate, that is, the particles stick together and continue on as a single larger particle, or they can fragment, the particles break apart into many smaller particles. The outcome of the collision depends on the relative velocity of the particles and their composition. At low velocities particles are expected to stick, while at high velocities collisions lead to fragmentation. The velocity that sets the boundary between the two outcomes is called the fragmentation velocity. For pure silicate aggregates the fragmentation velocities are low $\left(1 \mathrm{~m} \mathrm{~s}^{-1}\right)$ while aggregates with a coating of water ice can remain intact in collisions with relative velocities up to a order of magnitude higher (Blum \& Wurm 2008; Gundlach \& Blum 2015).

Dust size distributions resulting from the coagulation and fragmentation processes can generally not be computed analytically. Calculations have been done for both static and dynamic disks (Brauer et al. 2008; Birnstiel et al. 2010; Krijt \& Ciesla 2016). These models are very numerically intensive and do not lend themselves to the inclusion of additional physics and chemistry nor to large parameter studies. As such we will use the simplified dust evolution prescription from Birnstiel et al. (2012). This prescription has been benchmarked against models with a more complete grain growth and dust dynamics prescription from Brauer et al. (2008). The prescription only tracks the ends of the dust size distribution, the smallest grains of set size and the largest grains at a location in the disk of a variable size. As this prescription is a key part of the model we will reiterate some

2 Cratering and bouncing are neglected for simplicity. 
of the key arguments, equations and assumption of this prescription here, for a more complete explanation, see Birnstiel et al. (2012).

The prescription assumes that the dust size distribution can be in one of three stages at any point in the disk. Either the largest particles are still growing, the largest particle size is limited by radial drift, or the largest particle size is limited by fragmentation. In the first and second case, the size distribution is strongly biased towards larger sizes. In the final case the size distribution is a bit flatter (see, Brauer et al. 2008). The size distribution in all cases is parametrised by a minimal grain size, the monomer size, a maximal grain size, which depends on the local conditions, and the fraction of mass in the large grains.

From physical considerations one can write a maximal expected size of the particles due to different processes. Growth by coagulation is limited by the amount of collisions and thus by the amount of time that has passed. The largest grain expected in a size distribution at a given time is given by:

$a_{\text {grow }}(t)=a_{\text {mono }} \exp \left[\frac{t-t_{0}}{\tau_{\text {grow }}}\right]$,

where $a_{\text {mono }}$ is the monomer size, $t_{0}$ is the starting time, $t$ is the current time and $\tau_{\text {grow }}$ is the local growth time scale, given by:

$\tau_{\text {grow }}=\frac{\Sigma_{\text {gas }}}{\Sigma_{\text {dust }} \Omega}$.

Radial drift moves the particles inwards: the larger the particles, the faster the drift. There is thus a size at which particles are removed faster due to radial drift than they are replenished by grain growth, limiting the maximal size of particles. This maximal size is given by:

$a_{\mathrm{drift}}=f_{d} \frac{8 \Sigma_{\mathrm{dust}}}{\rho_{s}} \frac{\pi r^{2} \Omega^{2}}{c_{s}^{2}} \gamma^{-1}$,

with $f_{d}$ a numerical factor, $\rho_{s}$ is the density of the grains and $\gamma$ is the absolute power law slope of the gas pressure:

$\gamma=\left|\frac{\mathrm{d} \ln P_{\mathrm{gas}}}{\mathrm{d} \ln r}\right|$.

As mentioned before, particles that collide with high velocities are expected to fragment. For this model we consider two sources of relative velocities. One source of fragmentation is differential velocities due to turbulence (see, Ormel \& Cuzzi 2007, for a more complete explanation). This limiting size is given by:

$a_{\text {frag }}=f_{f} \frac{2}{3 \pi} \frac{\Sigma_{\text {gas }}}{\rho_{s} \alpha} \frac{u_{f}^{2}}{c_{s}^{2}}$,

where $f_{f}$ is a numerical fine tuning parameter and $u_{f}$ is the fragmentation velocity. The other source of fragmentation considered is different velocities due to different radial drift speeds. The limiting size for this process is given by:

$a_{\mathrm{df}}=\frac{u_{f} r \Omega}{c_{s}^{2}(1-N)} \frac{4 \Sigma_{\mathrm{gas}}}{\rho_{s}} \gamma^{-1}$,

$N$ is the average Stokes number fraction between two colliding grains, here we use $N=0.5$.

The size distribution at a location in the disk spans from the monomer size $\left(a_{\text {mono }}\right)$ to the smallest of the four limiting sizes above. In the model the grains size distribution is split into "small" and "large" grains. The mass fraction of the large grains depends on the process limiting the size: if the grain size is limited by fragmentation $\left(\min \left(a_{\mathrm{frag}}, a_{\mathrm{df}}\right)<a_{\mathrm{drift}}\right)$, the fraction of mass in large grains $\left(f_{m \text {, frag }}\right)$ is assumed to be $75 \%$. If the grain size is limited by radial drift $\left(a_{\mathrm{drift}}<\min \left(a_{\mathrm{frag}}, a_{\mathrm{df}}\right)\right)$, the fraction of mass in large grains $\left(f_{m \text {, drift }}\right)$ is assumed to be $97 \%$. These fractions were determined by Birnstiel et al. (2012) by matching the simplified model to more complete grain-growth models.

Using these considerations the advection-diffusion equation for the dynamics of the dust can be rewritten:

$$
\begin{aligned}
\frac{\partial \Sigma_{\text {dust }}}{\partial t}= & \frac{1}{r} \frac{\partial}{\partial r}\left[\left(u_{\text {dust }, \mathrm{s}} r\left(1-f_{m}\right) \Sigma_{\text {dust }}+u_{\text {dust }, 1} r f_{m} \Sigma_{\text {dust }}\right)\right. \\
& +r \Sigma_{\text {gas }}\left(D_{\text {dust }, \mathrm{s}} \frac{\partial}{\partial r}\left(\frac{\left(1-f_{m}\right) \Sigma_{\text {dust }}}{\Sigma_{\text {gas }}}\right)\right. \\
& \left.\left.+D_{\text {dust }, 1} \frac{\partial}{\partial r}\left(\frac{f_{m} \Sigma_{\text {dust }}}{\Sigma_{\text {gas }}}\right)\right)\right],
\end{aligned}
$$

where $D_{\text {dust, } \mathrm{x}}=D_{\text {gas }} /\left(1+\mathrm{St}_{\mathrm{x}}^{2}\right), u_{\text {dust, } \mathrm{x}}=u_{\text {gas }} /\left(1+\mathrm{St}_{\mathrm{x}}^{2}\right)+u_{\text {drift } \mathrm{x}}$ where $u_{\text {drift }}$ is the velocity due to radial drift (Eq. (10)). Here it is assumed that the large grains never get a Stokes number larger than unity, which holds for the models presented here.

The final part of the dynamics concerns the ice on the dust. The ice moves with the dust, so both the large scale movements as well as the mixing diffusion of dust must be taken into account. For the model we assume that the ice is distributed over the grains according to the mass of the grains. This means that if large grains have a mass fraction $f_{m}$, then the large grains will have the same mass fraction of $f_{m}$ of the available ice. This is a reasonable assumption, as long as the grain size distribution is in fragmentation equilibrium and the largest grains are transported on a timescale longer than the local growth timescale. The algorithm used here forces the latter condition to be true everywhere in the disk, while the former condition is generally true for the $\mathrm{CO}_{2}$ and $\mathrm{H}_{2} \mathrm{O}$ icelines (Brauer et al. 2008), but not for the CO iceline (Stammler et al. 2017). With this assumption the advection-diffusion equation for the ice can be written as:

$$
\begin{aligned}
\frac{\partial \Sigma_{\text {ice }, x}}{\partial t}= & \frac{1}{r} \frac{\partial}{\partial r} r\left(F_{\text {dust }} \frac{\Sigma_{\text {ice }, x}}{\Sigma_{\text {dust }}}\right. \\
& \left.+\Sigma_{\text {dust }}\left(D_{\text {dust }, \mathrm{s}}\left(1-f_{m}\right)+D_{\text {dust }, 1} f_{m}\right) \frac{\partial}{\partial r} \frac{\Sigma_{\text {ice }, \mathrm{x}}}{\Sigma_{\text {dust }}}\right) .
\end{aligned}
$$

Here $F_{\text {dust }}$ is the radial flux of dust, this is given by:

$$
\begin{aligned}
F_{\text {dust }}= & \Sigma_{\text {dust }}\left(u_{\text {dust }, s}\left(1-f_{m}\right)+u_{\text {dust }, 1} f_{m}\right) \\
& +\Sigma_{\text {dust }}\left(D_{\text {dust }, s} \frac{\partial}{\partial r}\left(\frac{\left(1-f_{m}\right) \Sigma_{\text {dust }}}{\Sigma_{\text {gas }}}\right)\right. \\
& \left.+D_{\text {dust }, 1} \frac{\partial}{\partial r}\left(\frac{f_{m} \Sigma_{\text {dust }}}{\Sigma_{\text {gas }}}\right)\right) .
\end{aligned}
$$

\subsection{Model parameters}

For our study, we pick a disk structure that should be representative of a young disk around a one solar mass star. The initial surface density structure is given by:

$\Sigma_{\text {gas }}(r)=\Sigma_{1 \mathrm{AU}}\left(\frac{r}{1 \mathrm{AU}}\right)^{-p} \exp \left[\left(-\frac{r}{r_{\text {taper }}}\right)^{2-p}\right]$, 
A. D. Bosman et al.: Efficiency of radial transport of ices: the case of $\mathrm{CO}_{2}$

where $p$ controls the steepness of the surface density profile, $r_{\text {taper }}$ the extent of the disk and $\Sigma_{1 \mathrm{AU}}$ sets the normalisation of the surface density profile. For our models we use $p=1$ and $r_{\text {taper }}=40 \mathrm{AU}$. The temperature profile is given by a simple power law:

$T(r)=T_{1 \mathrm{AU}}\left(\frac{r}{1 \mathrm{AU}}\right)^{-q}$.

The disk is assumed to be viscous with a constant $\alpha$, as such there is a constraint on the power law slopes $p$ and $q$, if we want the gas surface density to be a self similar solution to Eq. (1), it is required to have $p+q=3 / 2$ (Hartmann et al. 1998).

To calculate the volume densities, a vertical Gaussian distribution of gas with a scale height $H_{p}(r)=h_{p} r$, with $h_{p}=0.05$, is used.

The disk is populated with particles of $0.1 \mu \mathrm{m}$ at the start of the model, this is also the size of the small dust in the model. The gas-to-dust ratio is taken to be 100 over the entire disk. The density of the grains is assumed to be $2.5 \mathrm{~g} \mathrm{~cm}^{-3}$ and grains are assumed to be solid spheres.

The molecules are initially distributed through simple step functions. These step functions have a characteristic radius "iceline" which differentiates between the inner disk and the outer disk. Within this radius the gas phase abundance of the molecule is initialised, outside of this radius the solid phase abundance is initialised. Water is distributed equally through the disk with an abundance of $1.2 \times 10^{-4}$, whereas $\mathrm{CO}_{2}$ is initially depleted in the inner disk with an abundance of $10^{-8}$ (Pontoppidan et al. 2011; Bosman et al. 2017) and has a high abundance in the outer disk of $4 \times 10^{-5}$. This ice abundance of $\mathrm{CO}_{2}$ is motivated by the ISM ice abundance (Pontoppidan et al. 2008; Boogert et al. 2015), cometary abundances (Le Roy et al. 2015) and chemical models of disk formation (Drozdovskaya et al. 2016).

A summary of the initial conditions, fixed and variable parameters is given in Table 1 .

\subsection{Boundary conditions}

Due to finite computational capabilities, the calculation domain of the model needs to be limited. The assumptions made for the edges can have significant influences on the model results. For the inner edge, it is assumed that gas leaves the disk with an accretion rate as assumed from a self similar solution $(p+q=$ $3 / 2$ ) according to the viscosity and surface density at the inner edge. The accretion rate is given by:

$\dot{M}=3 \pi \Sigma_{\text {gas }} v_{\text {gas }}$.

All gas constituents advect with the gas over the inner boundary or, in the case of large grains, drift over the boundary. Diffusion over the inner boundary is not possible. For the outer boundary, the assumption is made that the surface density of gas outside the domain is zero. Again viscous evolution or an advection process can remove grains and other gas constituents from the computational domain.

\section{Chemical processes}

\subsection{Freeze-out and sublimation}

Freeze-out and sublimation determine the fraction of a molecule that is in the gas phase and the fraction that is locked up on the grains. Freeze-out of a molecule, or a molecule's accretion onto a grain is given by the collision rate of a molecule with the grain
Table 1. Initial conditions, fixed parameters and variables.

\begin{tabular}{|c|c|c|}
\hline Description & Symbol & Value \\
\hline \multicolumn{3}{|c|}{ Initial conditions and fixed parameters } \\
\hline \multicolumn{3}{|c|}{ Disk physical structure } \\
\hline Central stellar mass & $M_{\star}$ & $1 M_{\odot}$ \\
\hline Surface density at $1 \mathrm{AU}$ & $\Sigma_{1 \mathrm{AU}}$ & $15000 \mathrm{~kg} \mathrm{~m}^{-2}$ \\
\hline Temperature at $1 \mathrm{AU}$ & $T_{1 \mathrm{AU}}$ & $300 \mathrm{~K}$ \\
\hline Exponential taper radius & $r_{\text {taper }}$ & $40 \mathrm{AU}$ \\
\hline$\Sigma$ density power law slope & $p$ & 1 \\
\hline$T$ power law slope & $q$ & 0.5 \\
\hline Total initial disk mass & $M_{\mathrm{gas}, 0}$ & $0.02 M_{\odot}$ \\
\hline Disk FWHM angle & $h_{\mathrm{FWHM}}$ & $0.05 \mathrm{rad}$ \\
\hline \multicolumn{3}{|c|}{ Dust properties } \\
\hline Gas-to-dust ratio & $\mathrm{g} / \mathrm{d}$ & 100 \\
\hline Monomer size & $a_{\text {small }}$ & $0.1 \mu \mathrm{m}$ \\
\hline Grain density & $\rho_{s}$ & $2.5 \mathrm{~g} \mathrm{~cm}^{-3}$ \\
\hline Drift lim. large grain frac. & $f_{m, \text { drift }}$ & 0.97 \\
\hline Frag. lim. large grain frac. & $f_{m, \text { frag }}$ & 0.75 \\
\hline Num. factor fragmentation size & $f_{f}$ & 0.37 \\
\hline Num. factor drift size & $f_{d}$ & 0.55 \\
\hline \multicolumn{3}{|c|}{ Chemical parameters } \\
\hline Sticking fraction & $f_{s}$ & 1.0 \\
\hline Cosmic ray ionisation rate & $\zeta_{\mathrm{H}_{2}}$ & $10^{-17} \mathrm{~s}^{-1}$ \\
\hline Number of active ice layers & $N_{\text {act }}^{2}$ & 2 \\
\hline Step radius water & $r_{\text {step, } \mathrm{H}_{2} \mathrm{O}}$ & $3 \mathrm{AU}$ \\
\hline Inner disk $\mathrm{H}_{2} \mathrm{O}$ abundance & $x_{\mathrm{in}, \mathrm{H}_{2} \mathrm{O}}$ & $1.2 \times 10^{-4}$ \\
\hline Outer disk $\mathrm{H}_{2} \mathrm{O}$ abundance & $x_{\text {out }, \mathrm{H}_{2} \mathrm{O}}$ & $1.2 \times 10^{-4}$ \\
\hline $\mathrm{H}_{2} \mathrm{O}$ binding energy & $E_{\text {bind, } \mathrm{H}_{2} \mathrm{O}}$ & $5600 \mathrm{~K}$ \\
\hline $\mathrm{H}_{2} \mathrm{O}$ desorption prefactor & $p_{\mathrm{H}_{2} \mathrm{O}}$ & $10^{30} \mathrm{~cm}^{-2} \mathrm{~s}^{-1}$ \\
\hline $\mathrm{H}_{2} \mathrm{O} C R$ destruction efficiency & $k_{\mathrm{H}_{2} \mathrm{O}} / \zeta_{\mathrm{H}_{2}}$ & 1800 \\
\hline Step radius $\mathrm{CO}_{2}$ & $r_{\text {step, } \mathrm{CO}_{2}}$ & $10 \mathrm{AU}$ \\
\hline Inner disk $\mathrm{CO}_{2}$ abundance & $x_{\mathrm{in}, \mathrm{CO}_{2}}$ & $1 \times 10^{-8}$ \\
\hline Outer disk $\mathrm{CO}_{2}$ abundance & $x_{\mathrm{out}, \mathrm{CO}_{2}}$ & $4 \times 10^{-5}$ \\
\hline $\mathrm{CO}_{2}$ binding energy & $E_{\text {bind, } \mathrm{CO}_{2}}$ & $2900 \mathrm{~K}$ \\
\hline $\mathrm{CO}_{2}$ desorption prefactor & $p_{\mathrm{CO}_{2}}$ & $9.3 \times 10^{26} \mathrm{~cm}^{-2} \mathrm{~s}^{-1}$ \\
\hline \multicolumn{3}{|c|}{ Variables } \\
\hline Viscosity parameter & $\alpha$ & \\
\hline Fragmentation velocity & $u_{\mathrm{f}}$ & \\
\hline $\mathrm{CO}_{2}$ destruction rate & $R_{\text {destr, } \mathrm{CO}_{2}}$ & \\
\hline
\end{tabular}

surface times the sticking fraction, $f_{s}$, which is assumed to be unity:

$R_{\mathrm{acc}, \mathrm{x}}=f_{s} \sigma_{\text {dust }} n_{\text {grain }} v_{\text {therm }, \mathrm{x}}$,

where $\sigma_{\text {dust }}$ is the average dust surface area, $n_{\text {grain }}$ is the number density of grains and the thermal velocity $v_{\text {therm,x }}=\sqrt{8 k T / \pi m_{x}}$, with $k$ the Boltzmann constant, $T$ the temperature and $m_{x}$ the mass of the molecule. Molecules that are frozen-out on the grain can sublimate or desorb. For a grain covered with many monolayers of ice, the rate per unit volume for this process is given by:

$f_{\text {des }, \mathrm{x}}=R_{\text {des }, \mathrm{x}} n_{\mathrm{x}, \text { ice }}=p_{x} \sigma_{\text {dust }} n_{\text {grain }} N_{\text {act }} \exp \left[-\frac{E_{\text {bind }}}{k T}\right]$,

where $p_{x}$ is the zeroth-order "prefactor" encoding the frequency of desorption attempts per unit surface area, $\sigma_{\text {dust }}$ is the surface area per grain, $n_{\text {grain }}$ is the number density of grains. $N_{\text {act }}$ is the number of active layers, that is, the number of layers that can participate in the sublimation, $N_{\text {act }}=2$ is used, $T$ is the dust temperature, which we take equal to the gas temperature. 
For mixed ices this rate can be modified by a covering fraction $\chi_{x}=n_{\text {ice, } x} / \sum_{x} n_{\text {ice, } x}$, however, this is neglected here. We note that $f_{\text {des, } x}$ in its current form is independent of the amount of molecules frozen out on the dust grains. Using these rates we get the following differential equation:

$\frac{\partial n_{\mathrm{x}, \mathrm{gas}}}{\partial t}=-R_{\mathrm{acc}, \mathrm{x}} n_{\mathrm{x}, \mathrm{gas}}+f_{\mathrm{des}, \mathrm{x}}$,

which has an analytical solution:

$$
\begin{aligned}
n_{\mathrm{x}, \mathrm{gas}}(t)= & \min \left[n_{\mathrm{x}, \mathrm{tot}},\left(n_{\mathrm{x}, \mathrm{gas}}\left(t_{0}\right)-\frac{f_{\mathrm{des}, \mathrm{x}}}{R_{\mathrm{acc}, \mathrm{x}}}\right)\right. \\
& \left.\times \exp \left(-R_{\mathrm{acc}, \mathrm{x}}\left(t-t_{0}\right)\right)+\frac{f_{\mathrm{des}, \mathrm{x}}}{R_{\mathrm{acc}, \mathrm{x}}}\right] .
\end{aligned}
$$

where $n_{\mathrm{x}, \text { tot }}$ is the total number density of a molecule (gas and ice). The number density of ice is given by:

$n_{\mathrm{x}, \text { ice }}(t)=n_{\mathrm{x}, \mathrm{tot}}-n_{\mathrm{x}, \mathrm{gas}}(t)$.

The ice line temperature, defined as the temperature for which $n_{\mathrm{x}, \mathrm{gas}}=n_{\mathrm{x} \text {,dust }}$, that is, when freeze-out and sublimation balance, depends on the total number density of the molecule considered. At lower total molecule number densities, the iceline will be at lower temperatures.

For $\mathrm{CO}_{2}$ we use a binding energy of $2900 \mathrm{~K}$, representative for $\mathrm{CO}_{2}$ mixed with water (Sandford \& Allamandola 1990; Collings et al. 2004). More recent measurements have suggested that the binding energy is lower, around $2300 \mathrm{~K}$ (Noble et al. 2012). Using the lower binding energy moves the $\mathrm{CO}_{2}$ iceline further out from 90 to $80 \mathrm{~K}$ or from 6 to $8 \mathrm{AU}$ in our standard model. The prefactor, $p_{x, \mathrm{CO}_{2}}$, of $9.3 \times 10^{26} \mathrm{~cm}^{-2} \mathrm{~s}^{-1}$ from (Noble et al. 2012) is used. The change in iceline location has a minimal effect on the evolution of the abundance profiles. The mixing time-scale becomes longer at larger radii which would increase mixing times. For $\mathrm{H}_{2} \mathrm{O}$ a binding energy of $5600 \mathrm{~K}$ and prefactor, $p_{x, \mathrm{H}_{2} \mathrm{O}}$, of $10^{30} \mathrm{~cm}^{-2} \mathrm{~s}^{-1}$ (Fraser et al. 2001).

\subsection{Midplane formation and destruction processes}

Radial drift and radial diffusion and advection will quickly move part of the outer disk $\mathrm{CO}_{2}$ ice reservoir into the inner disk, enhancing the inner disk abundances. To get a good measure of the amount of $\mathrm{CO}_{2}$ in the inner disk it is necessary to also take into account the processes that form and destroy $\mathrm{CO}_{2}$ in gas and ice. The density is highest near the mid-plane, as such this is where formation and destruction process are expected to be most relevant for the bulk of the $\mathrm{CO}_{2}$. However, in the less dense upper layers, there are UV-photons that can dissociate and ionise molecules, possibly influencing the overall abundance of $\mathrm{CO}_{2}$.

\subsubsection{Gas-phase formation of $\mathrm{CO}_{2}$}

The formation of $\mathrm{CO}_{2}$ in the inner disk mainly goes through the warm gas-phase route. Here $\mathrm{CO}_{2}$ forms through the reaction $\mathrm{CO}+\mathrm{OH} \longrightarrow \mathrm{CO}_{2}+\mathrm{H}$. The reaction has a slight activation barrier of $176 \mathrm{~K}$ (Smith et al. 2004). The parent molecule CO is very stable and is expected to be present at high abundances in the inner disk $\left(10^{-4}\right)$ (Walsh et al. 2015). The $\mathrm{OH}$ radical is expected to be less abundant, and it is the fate of this radical that determines the total production rate of $\mathrm{CO}_{2} . \mathrm{OH}$ is formed either directly from $\mathrm{H}_{2} \mathrm{O}$ photodissociation (Heays et al. 2017), or by hydrogenation of atomic oxygen, $\mathrm{O}+\mathrm{H}_{2} \longrightarrow \mathrm{OH}+\mathrm{H}$, in

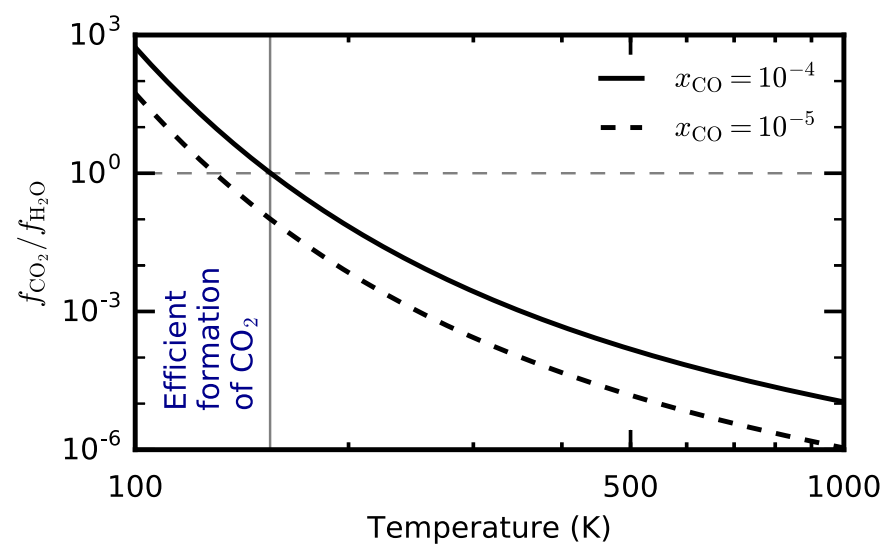

Fig. 1. Ratio of the $\mathrm{CO}_{2}$ to $\mathrm{H}_{2} \mathrm{O}$ formation rate from a reaction of $\mathrm{OH}$ with $\mathrm{CO}$ and $\mathrm{H}_{2}$ respectively. Hydrogenation of $\mathrm{OH}$ to $\mathrm{H}_{2} \mathrm{O}$ dominates above $150 \mathrm{~K}$. Formation times of $\mathrm{CO}_{2}$ and $\mathrm{H}_{2} \mathrm{O}$ from $\mathrm{OH}$ are faster than the inner disk mixing time for all temperatures considered here.

a reaction that has an activation barrier of $3150 \mathrm{~K}$ (Baulch et al. 1992). The atomic oxygen itself also has to be liberated from, in this case, either $\mathrm{CO}, \mathrm{CO}_{2}$ or $\mathrm{H}_{2} \mathrm{O}$ by X-rays or UV-photons. The production rate of $\mathrm{CO}_{2}$ is thus severely limited if there is no strong radiation field present to release oxygen from one of the major carriers.

The $\mathrm{CO}_{2}$ formation reaction, $\mathrm{CO}+\mathrm{OH} \longrightarrow \mathrm{CO}_{2}+\mathrm{H}$, has competition from the $\mathrm{H}_{2} \mathrm{O}$ formation reaction, $\mathrm{OH}+\mathrm{H}_{2} \longrightarrow$ $\mathrm{H}_{2} \mathrm{O}+\mathrm{H}$. The hydrogenation of $\mathrm{OH}$ has a higher activation energy, $1740 \mathrm{~K}$, than the formation of $\mathrm{CO}_{2}$, but since $\mathrm{H}_{2}$ is orders of magnitude more abundant than $\mathrm{CO}$, the formation of water will dominate over the formation of $\mathrm{CO}_{2}$ at high temperatures. The rate for $\mathrm{CO}_{2}$ formation is given by (Smith et al. 2004):

$f_{\text {form }, \mathrm{CO}_{2}}=2.81 \times 10^{-13} n_{\mathrm{CO}} n_{\mathrm{OH}} \exp \left(-\frac{176 \mathrm{~K}}{T}\right)$.

The formation rate for $\mathrm{H}_{2} \mathrm{O}$ formation is given by (Baulch et al. 1992):

$f_{\text {form }, \mathrm{H}_{2} \mathrm{O}}=2.05 \times 10^{-12} n_{\mathrm{H}_{2}} n_{\mathrm{OH}}\left(\frac{T}{300 \mathrm{~K}}\right)^{1.52} \exp \left(-\frac{1740 \mathrm{~K}}{T}\right)$.

This means that the expected $x_{\mathrm{CO}_{2}} / x_{\mathrm{H}_{2} \mathrm{O}}$ fraction from formation is:

$\frac{f_{\text {form }, \mathrm{CO}_{2}}}{f_{\text {form }, \mathrm{H}_{2} \mathrm{O}}}=0.14 x_{\mathrm{CO}}\left(\frac{T}{300 \mathrm{~K}}\right)^{-1.52} \exp \left(\frac{1564 \mathrm{~K}}{T}\right)$.

This function is plotted in Fig. 1 which shows that the formation of $\mathrm{CO}_{2}$ is faster below temperatures of $150 \mathrm{~K}$, whereas above this temperature formation of water is faster. Above a temperature of $300 \mathrm{~K}$ water formation is a thousand times faster than $\mathrm{CO}_{2}$ formation. The implication is that gaseous $\mathrm{CO}_{2}$ formation is only effective in a narrow temperature range, $50-150 \mathrm{~K}$, and then only if $\mathrm{OH}$ is present as well, requiring UV photons or X-rays to liberate $\mathrm{O}$ and $\mathrm{OH}$ from $\mathrm{CO}$ or $\mathrm{H}_{2} \mathrm{O}$.

\subsubsection{Destruction of $\mathrm{CO}_{2}$ : cosmic rays}

Cosmic rays, $10-100 \mathrm{MeV}$ protons and ions, have enough energy to penetrate deeply into the disk. Cosmic-rays can ionise $\mathrm{H}_{2}$ in 
A. D. Bosman et al.: Efficiency of radial transport of ices: the case of $\mathrm{CO}_{2}$

regions where UV photons and X-rays cannot penetrate. The primary ionisation as well as the collisions of the resulting energetic electron with further $\mathrm{H}_{2}$ creates electronically excited $\mathrm{H}_{2}$ as well as excited $\mathrm{H}$ atoms. These excited atoms and molecules radiatively decay, resulting in the emission of UV-photons (Prasad \& Tarafdar 1983). These locally generated UV-photons can dissociate and ionise molecules. Here only the dissociation rate of $\mathrm{CO}_{2}$ is taken into account as ionisation of $\mathrm{CO}_{2}$ by this process is negligible. Following Heays et al. (2017) the destruction rate of species $X$ is written as:

$k_{X}=\frac{\zeta_{\mathrm{H}_{2}} x_{\mathrm{H}_{2}}}{x_{X}} \int P(\lambda) p_{X}(\lambda) \mathrm{d} \lambda$,

where $\zeta_{\mathrm{H}_{2}}$ is the direct cosmic ray ionisation rate of $\mathrm{H}_{2}, x_{X}$ is the abundance of species $X$ w.r.t. $\mathrm{H}_{2}, P(\lambda)$ is the photon emission probability per unit spectral density for which we use the spectrum from Gredel et al. (1987). $p_{X}(\lambda)$ is the absorption probability of species $X$ for a photon of wavelength $\lambda$. This probability is given by:

$p_{X}(\lambda)=\frac{x_{X} \sigma_{X}^{\text {destr }}(\lambda)}{x_{\text {dust }} \sigma_{\text {dust }}^{\text {abs }}(\lambda)+\sum_{j} x_{j} \sigma_{j}^{\text {abs }}(\lambda)}$,

where $\sigma_{X}^{\mathrm{i}}(\lambda)$ is the wavelength dependent destruction or absorption cross section of species $X$ and $x_{\text {dust }} \sigma_{\text {dust }}^{\text {abs }}$ is the dust cross section per hydrogen molecule. For the calculation of the cosmic-ray dissociation rate of $\mathrm{CO}_{2}$ we assume that the destruction cross section in the UV is equal to the absorption cross section for $\mathrm{CO}_{2}$, that is, every absorption of a photon with a wavelength shorter than $227 \mathrm{~nm}$ leads to the destruction of a $\mathrm{CO}_{2}$ molecule. For the calculations the cross sections from Heays et al. (2017) are used ${ }^{3}$. These cross sections can also be used to compute destruction rates for $\mathrm{CO}_{2}$ by stellar UV radiation in the surface layers of the disk.

The dust absorption is an important factor in these calculations and can be the dominant contribution to the total absorption in parts of the spectrum. The dust absorption greatly depends on the dust opacities assumed. A standard ISM dust composition was taken following Weingartner \& Draine (2001), the mass extinction coefficients are calculated using Mie theory with the MIEX code (Wolf \& Voshchinnikov 2004) and optical constants by Draine (2003) for graphite and Weingartner \& Draine (2001) for silicates. Grain sizes are distributed assuming an MRN distribution starting at $5 \mathrm{~nm}$, with varying maximum size are used. The resulting mass opacities and cross sections are shown in Fig. A.1.

Cosmic ray induced destruction rates for $\mathrm{CO}_{2}$ are calculated for each dust size distribution for a range of $\mathrm{CO}_{2}$ abundances between $10^{-8}$ and $10^{-4}$. The o-p ratio of $\mathrm{H}_{2}$, important for the $\mathrm{H}_{2}$ emission spectrum, is assumed to be 3:1, representative for high temperature gas. The abundances of the other shielding species used in the calculation are shown in Table 2. The destruction rate for $\mathrm{CO}_{2}$ is plotted in Fig. 2.

The $\mathrm{CO}_{2}$ destruction is faster for grown grains and low abundances of $\mathrm{CO}_{2}$. At abundances above $10^{-7}$, the strongest transitions start to saturate, lowering the destruction rate with increasing abundance. Even though the dust opacity changes by more than an order of magnitude, the dissociation rates stay within a factor of 3 for all $\mathrm{CO}_{2}$ abundances. This is due to the inclusion of $\mathrm{H}_{2} \mathrm{O}$ into our calculations. $\mathrm{H}_{2} \mathrm{O}$ has large absorption

3 UV cross-sections can be found here:

http://home.strw.leidenuniv.nl/ ewine/photo/
Table 2. Gas-phase abundances assumed for the cosmic ray induced dissociation rate calculations.

\begin{tabular}{lll}
\hline \hline Molecule & $\begin{array}{l}\text { Abundance } \\
\text { inside } \mathrm{H}_{2} \mathrm{O} \text { iceline }\end{array}$ & $\begin{array}{l}\text { Abundance } \\
\text { outside } \mathrm{H}_{2} \mathrm{O} \text { iceline }\end{array}$ \\
\hline $\mathrm{H}_{2}$ & 1 & 1 \\
$\mathrm{H}$ & $10^{-12}$ & $10^{-10}$ \\
$\mathrm{CO}$ & $10^{-5}$ & $10^{-5}$ \\
$\mathrm{~N}_{2}$ & $10^{-5}$ & $10^{-5}$ \\
$\mathrm{CO}_{2}$ & $10^{-8 \cdots-4}$ & $10^{-8 \cdots-4}$ \\
$\mathrm{H}_{2} \mathrm{O}$ & $10^{-4}$ & $10^{-8}$ \\
\hline
\end{tabular}

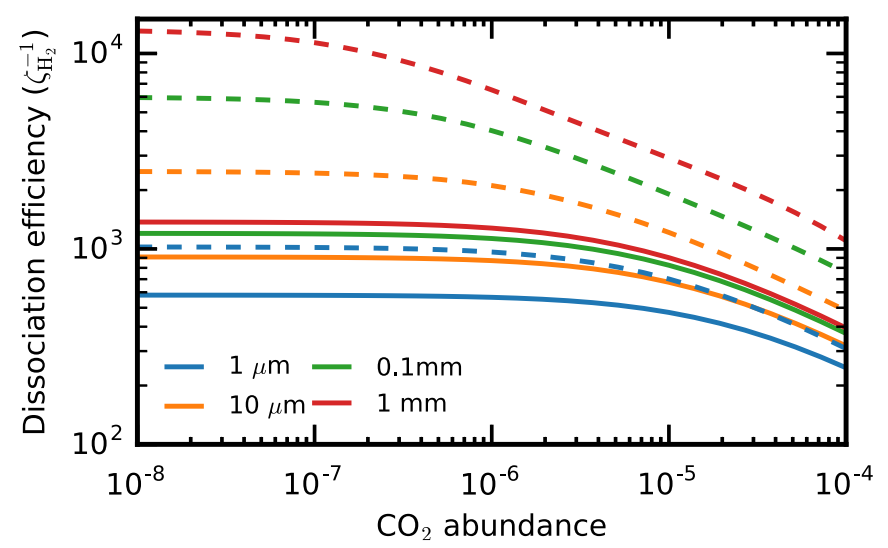

Fig. 2. Dissociation rate of $\mathrm{CO}_{2}$ due to cosmic ray induced photons, for different dust size distributions. Solid lines are for condition inside of the $\mathrm{H}_{2} \mathrm{O}$ iceline, dashed lines for conditions outside of the $\mathrm{H}_{2} \mathrm{O}$ iceline. The efficiency multiplied by $\zeta_{\mathrm{H}_{2}}$ gives the $\mathrm{CO}_{2}$ dissociation rate.

cross sections in the same wavelength regimes as $\mathrm{CO}_{2}$. When $\mathrm{H}_{2} \mathrm{O}$ is depleted, such as would be expected between the $\mathrm{H}_{2} \mathrm{O}$ and $\mathrm{CO}_{2}$ icelines, $\mathrm{CO}_{2}$ destruction rates increase, especially for the largest grains. Even in this optimal case, the destruction rate for $\mathrm{CO}_{2}$ is limited to $2 \times 10^{-13} \mathrm{~s}^{-1}$ for typical $\zeta_{\mathrm{H}_{2}}$ of $10^{-17} \mathrm{~s}^{-1}$.

Aside from generating a UV field, cosmic-rays also create ions, the most important of these being $\mathrm{He}^{+}$. Due to the large ionisation potential of $\mathrm{He}$, electron transfer reactions with $\mathrm{He}^{+}$ generally lead to dissociation of the newly created ion. For example, $\mathrm{CO}_{2}+\mathrm{He}^{+}$preferably leads to the creation of $\mathrm{O}+\mathrm{CO}^{+}$. Destruction of $\mathrm{CO}_{2}$ due to $\mathrm{He}^{+}$is limited by the creation of $\mathrm{He}^{+}$and the competition between $\mathrm{CO}_{2}$ and other reaction partners of $\mathrm{He}^{+}$. The total reaction rate coefficient of $\mathrm{CO}_{2}$ with $\mathrm{He}^{+}$ is $k_{\text {ion, } \mathrm{CO}_{2}}=1.14 \times 10^{-9} \mathrm{~cm}^{3} \mathrm{~s}^{-1}$ (Adams \& Smith 1976). The three main competitors for reactions with $\mathrm{He}^{+}$are $\mathrm{H}_{2} \mathrm{O}, \mathrm{CO}$ and $\mathrm{N}_{2}$, with reaction rate coefficients of $k_{\text {ion, } \mathrm{H}_{2} \mathrm{O}}=5.0 \times 10^{-10}$, $k_{\text {ion, } \mathrm{CO}}=1.6 \times 10^{-9}$ and $k_{\text {ion, } \mathrm{N}_{2}}=1.6 \times 10^{-9} \mathrm{~cm}^{3} \mathrm{~s}^{-1}$ respectively (Mauclaire et al. 1978; Anicich et al. 1977; Adams \& Smith 1976). Assuming a cosmic $\mathrm{He}^{+}$ionisation rate of $0.65 \zeta_{\mathrm{H}_{2}}$ (Umebayashi \& Nakano 2009), the destruction rate for $\mathrm{CO}_{2}$ due to $\mathrm{He}^{+}$reaction can be written as,

$R_{\text {destr, } \mathrm{He}^{+}}=0.65 \zeta_{\mathrm{H}_{2}} \frac{x_{\mathrm{He}}}{x_{\mathrm{CO}_{2}}} \frac{k_{\mathrm{ion}, \mathrm{CO}_{2}} x_{\mathrm{CO}_{2}}}{\sum_{X} k_{\mathrm{ion}, \mathrm{X}} x_{X}}$,

where the sum is over all reactive collision partners of $\mathrm{He}^{+}$of which $\mathrm{CO}, \mathrm{N}_{2}$ and $\mathrm{H}_{2} \mathrm{O}$ are most important. For typical abundances of $\mathrm{He}$ of 0.1 and $\mathrm{N}_{2}, \mathrm{CO}$ and $\mathrm{H}_{2} \mathrm{O}$ of $10^{-4}, R_{\text {destr, } \mathrm{He}^{+}}$is below $650 \zeta_{\mathrm{H}_{2}}$ for all $\mathrm{CO}_{2}$ abundances. Thus cosmic-ray induced 
photodissociation will always be more effective than destruction due to $\mathrm{He}^{+}$.

Altogether the destruction time-scale for midplane $\mathrm{CO}_{2}$ by cosmic ray induced processes is long, $\sim 3 \mathrm{Myr}$ for a $\zeta_{\mathrm{H}_{2}}=$ $10^{-17} \mathrm{~s}^{-1}$. The latter value is likely an upper limit for the inner disk given the possibility of attenuation and exclusion of cosmic rays (Umebayashi \& Nakano 1981; Cleeves et al. 2015).

\subsubsection{Destruction of $\mathrm{CO}_{2}$ : gas-phase reactions}

In warm gas it is possible to destroy $\mathrm{CO}_{2}$ by endothermic reactions with $\mathrm{H}$ or $\mathrm{H}_{2}$ (Talbi \& Herbst 2002). The reaction

$\mathrm{CO}_{2}+\mathrm{H} \longrightarrow \mathrm{CO}+\mathrm{OH}$

has an activation barrier of $13300 \mathrm{~K}$ and a pre-exponential factor of $2.5 \times 10^{-10} \mathrm{~cm}^{3} \mathrm{~s}^{-1}$ for temperatures between 300 and $2500 \mathrm{~K}$ (Tsang \& Hampson 1986) while the reaction

$\mathrm{CO}_{2}+\mathrm{H}_{2} \longrightarrow \mathrm{CO}+\mathrm{H}_{2} \mathrm{O}$

has an activation barrier of $56900 \mathrm{~K}$ and has a pre-exponential factor of $3.3 \times 10^{-10} \mathrm{~cm}^{3} \mathrm{~s}^{-1}$ at $1000 \mathrm{~K}$. This means that for gas at $300 \mathrm{~K}$, an $\mathrm{H}_{2}$ density of $10^{12} \mathrm{~cm}^{-3}$, and a corresponding $\mathrm{H}$ density of $1 \mathrm{~cm}^{-3}$, the rate for destruction by atomic hydrogen is $1.4 \times 10^{-29} \mathrm{~s}^{-1}$, while the destruction rate by molecular hydrogen is $1.4 \times 10^{-80} \mathrm{~s}^{-1}$. Both are far too low to be significant in the inner disk. However, if the atomic $\mathrm{H}$ abundance is higher, destruction of $\mathrm{CO}_{2}$ by $\mathrm{H}$ can become efficient at high temperatures. As such the destruction of $\mathrm{CO}_{2}$ becomes very dependent on the formation speed of $\mathrm{H}_{2}$ at high densities and temperatures. The $\mathrm{CO}_{2}$ abundance as a function of temperature for a gas-phase model is shown in Fig. B.1. Temperatures of $>700 \mathrm{~K}$ are needed to lower the $\mathrm{CO}_{2}$ abundance below $10^{-7}$, even with a high atomic $\mathrm{H}$ abundance.

\subsection{Simulating spectra}

To compare the model abundances with observations, infrared spectra are simulated with the thermochemical code DALI (Bruderer et al. 2012; Bruderer 2013). DALI is used to calculate a radial temperature profile, from dust and gas surface density profiles and stellar parameters. The viscous evolution model is initialised using the same surface density distribution as the DALI model. The temperature slope $q$ is taken so that the surface density slope $p$ is consistent with a self-similar solution $q=1.5-p=0.9$. The temperature at $1 \mathrm{AU}$ is taken from the midplane temperatures as calculated by the DALI continuum ray-tracing module. No explicit chemistry is included in this version of DALI, but the abundances are parametrised using the output of the dynamical model. The viscous model run with $\alpha=10^{-3}, u_{f}=3 \mathrm{~m} \mathrm{~s}^{-1}$ and $\mathrm{CO}_{2}$ destruction rate varying between $10^{-13}$ and $10^{-9} \mathrm{~s}^{-1}$. The results from Sect. 4.4 show that these parameters represent the bulk of the model results.

After simulating $1 \mathrm{Myr}$ of evolution, the gas-phase $\mathrm{CO}_{2}$ abundance profile is extracted as function of temperature and interpolated onto the DALI midplane temperatures. The abundance is taken to be vertically constant up to the point where $A_{\mathrm{V}}=1$. In some variations an abundance floor of $10^{-9}$ or $10^{-8}$ is used for cells with $A_{\mathrm{V}}>1$ to simulate local $\mathrm{CO}_{2}$ production. Using the resulting abundance structure, the non-LTE excitation of $\mathrm{CO}_{2}$ is calculated using the rate coefficients from Bosman et al. (2017). Finally the DALI line ray-tracing module calculates the line fluxes for $\mathrm{CO}_{2}$ and its ${ }^{13} \mathrm{C}$ isotope.
Table 3. Adopted standard model parameters for the DALI modelling.

\begin{tabular}{llc}
\hline \hline Parameter & & Value \\
\hline Star & & \\
Mass & $M_{\star}\left[M_{\odot}\right]$ & 1.0 \\
Luminosity & $L_{\star}\left[L_{\odot}\right]$ & 4.0 \\
Effective temperature & $T_{\text {eff }}[\mathrm{K}]$ & 4250 \\
Accretion luminosity & $L_{\text {accr }}\left[L_{\odot}\right]$ & 3.3 \\
Accretion temperature & $T_{\text {accr }}[\mathrm{K}]$ & 10000 \\
\hline Disk & & \\
Dustdisk mass & $M_{\text {dust }}\left[M_{\odot}\right]$ & $2.9 \times 10^{-4}$ \\
Surface density index & $p$ & 0.9 \\
Characteristic radius & $R_{c}[\mathrm{AU}]$ & 46 \\
Inner radius & $R_{\text {in }}[\mathrm{AU}]$ & 0.19 \\
Scale height index ${ }^{b}$ & $\psi$ & 0.11 \\
Scale height angle ${ }^{b}$ & $h_{c}[\mathrm{rad}]$ & 0.18 \\
\hline DALI dust properties ${ }^{a}$ & & \\
Size & $a[\mu \mathrm{m}]$ & $0.005-1000$ \\
Size distribution & & $\mathrm{d} n / \mathrm{d} a \propto a^{-3.5}$ \\
Composition & & ISM \\
Gas-to-dust ratio & & 1000 \\
\hline Distance & $d$ [pc] $^{\circ}$ & 125 \\
Inclination & $\left.i{ }^{\circ}\right]$ & 20 \\
\hline${ }^{12} \mathrm{CO}_{2}{ }^{13}{ }^{13} \mathrm{CO}_{2}$ ratio & & 69 \\
\hline
\end{tabular}

Notes. ${ }^{(a)}$ Dust properties are the same as those used in Andrews et al. (2009) and Bruderer et al. (2015). Dust composition and optical constants are taken from Draine \& Lee (1984) and Weingartner \& Draine (2001). ${ }^{(b)}$ Only used in the DALI model, not in the viscous disk model. The viscous disk model assumes a geometrically flat disk.

For calculating the spectra, the same disk model is used as in Bruderer et al. (2015) and Bosman et al. (2017). The model is based on the disk AS $205 \mathrm{~N}$. The parameters for the DALI models can be found in Table 3. For more specifics on the modelling of the spectra and adopted parameters, see Bruderer et al. (2015) and Bosman et al. (2017). To simulate the high gas-todust ratios that are inferred from water observations (Meijerink et al. 2009), the overall gas-to-dust ratio is set at 1000 throughout the disk. The artificially high gas-to-dust ratio does not affect any of the modelling, except for the line formation, which is only sensitive to the upper optically thin layers of the disk. High gasto-dust ratios effectively mimic settled dust near the midplane containing $\sim 90 \%$ of the dust mass.

\section{Results}

\subsection{Pure viscous evolution}

To start, a dynamical model without grain growth and without any chemistry (except for freeze-out and desorption) is investigated. Figure 3 shows the time evolution for a model with $\alpha=10^{-3}$. As expected, the total gas and dust surface densities barely change over $10^{6} \mathrm{yr}$. The gas and dust evolve viscously, some mass is accreted onto the star while the outer disk spreads a little bit. There are no changes in gas-to-dust ratio in the disk.

The water abundance in the right panel of Fig. 3 shows no evolution at all. This is to be expected because the only radial evolution is due to the gas viscosity, which affects gas and dust equally. Diffusion of the icy dust grains is equally quick as the diffusion of the water vapour. This means that all the water vapour that diffuses outwards is compensated by icy dust grains diffusing inwards. 

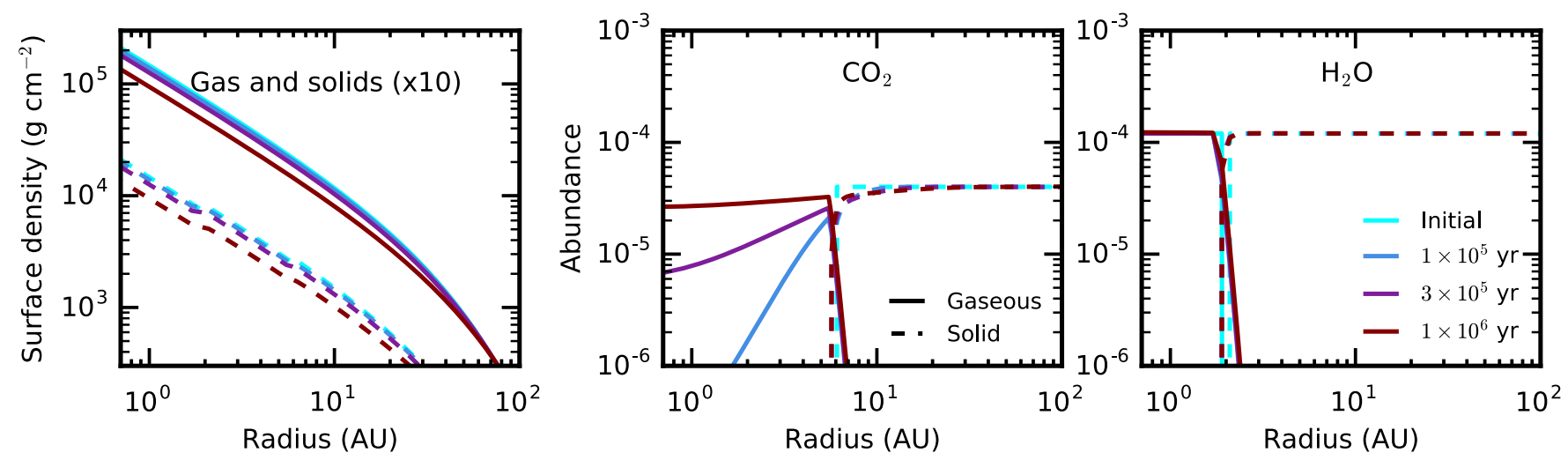

Fig. 3. Time evolution series for a model without grain growth. This model assumes an $\alpha$ of $10^{-3}$. Left: surface density of the gas (solid lines) and solids (dashed lines), the solid surface density is the sum of the dust and ice surface densities, it has been multiplied by a factor of 10 for visualization. Middle: abundance of $\mathrm{CO}_{2}$. Right: abundance of $\mathrm{H}_{2} \mathrm{O}$.

The abundance of $\mathrm{CO}_{2}$ in the middle panel of Fig. 3 does show evolution. Initially there is only a little bit of $\mathrm{CO}_{2}$ gas in the inner disk, but a large amount of ice in the outer disk. The abundance gradient together with the viscous accretion makes the icy $\mathrm{CO}_{2}$ move inwards, filling up the inner disk with $\mathrm{CO}_{2}$ gas. This continues for about $1 \times 10^{6} \mathrm{yr}$ until the gaseous abundance of $\mathrm{CO}_{2}$ is equal to the abundance of icy $\mathrm{CO}_{2}$. This time-scale directly scales with the assumed $\alpha$ parameter. For $\alpha=10^{-4}$ it takes more than $3 \mathrm{Myr}$ to get a flat abundance profile in the inner disk.

\subsection{Viscous evolution and grain growth}

For the models including grain growth, we tested a range of fragmentation velocities from 1 to $30 \mathrm{~m} \mathrm{~s}^{-1}$. For some of these models, especially those with low fragmentation velocities and high $\alpha$, the results are indistinguishable from the case without grain growth. Figures 4 and 5 show two models where the effect of grain growth and resulting drift can be seen.

Figure 4 shows the surface density and abundance evolution for a model with a fragmentation velocity of $3 \mathrm{~m} \mathrm{~s}^{-1}$ as appropriate for pure silicate grains. The surface density of the dust shows a small evolution due to radial drift. Instead of decreasing, the surface density of dust in the inner 4 AU slightly increases in the first $300000 \mathrm{yr}$ due to the supply of dust particles from outside this radius.

The abundance profiles in Fig. 4 show distinct effects of radial drift. Both the gaseous $\mathrm{H}_{2} \mathrm{O}$ and $\mathrm{CO}_{2}$ abundances are high at all times due to the influx of drifting icy grains. There is also a decrease in the abundance of ices at large radii. This is because the grains carrying the ices have moved inwards, increasing the gas-to-ice ratios.

The models are not in steady state after $1 \mathrm{Myr}$. If these models are evolved further, at first the abundances of $\mathrm{H}_{2} \mathrm{O}$ and $\mathrm{CO}_{2}$ will increase further. At some point in time the influx of dust will slow down, because a significant part of the dust in the outer disk will have drifted across the snowline. At this point the inner disk abundances of $\mathrm{H}_{2} \mathrm{O}$ and $\mathrm{CO}_{2}$ start to decrease as these molecules are lost due to accretion onto the star but no longer replenished by dust from the outer disk. For the model shown in Fig. 4 the average gas-to-dust ratio in the disk would be around 500 after 3 Myr.

For higher $\alpha$ the effects of radial drift are limited as the high rate of mixing smooths out concentration gradients created by radial drift. At the same time the maximum grain size is limited due to larger velocity collisions at higher turbulence. As such, for $\alpha=0.01$ a fragmentation velocity higher than $10 \mathrm{~m} \mathrm{~s}^{-1}$ is needed to see significant effects of radial drift and grain growth.

For lower $\alpha$ the effects of grain growth get more pronounced, $90 \%$ of the dust mass is accreted onto the star in $2.5 \mathrm{Myr}$ for a fragmentation velocity of $1 \mathrm{~m} \mathrm{~s}^{-1}$. In this time a lot of molecular material is released into the inner disk and peak abundances of $10^{-3}$ and $10^{-2}$ are reached for $\mathrm{CO}_{2}$ and $\mathrm{H}_{2} \mathrm{O}$, respectively, after $1 \mathrm{Myr}$ of evolution. Due to the low turbulence in the gas, the volatiles released are not well mixed, neither inwards nor outwards, so there is no strong enhancement of the ice surface density just outside of the iceline. An overview of the different model evolutions can be found in Appendix C.

Increasing the fragmentation velocity allows grains in the model to grow to larger sizes, leading again to larger amounts of radial drift. Figure 5 shows the evolution of the same model as shown in Fig. 4, but with a fragmentation velocity of $30 \mathrm{~m} \mathrm{~s}^{-1}$, representative for grains coated in water ice. The solid surface density distribution clearly shows the effects of grain growth. At all radii, mass is moved inwards at a high rate. When the silicate surface density has dropped by an order of magnitude, a local enhancement in the surface density of the total solids is seen at the icelines. This enhancement is about a factor of 2 for both icelines.

The gas-phase abundances of $\mathrm{CO}_{2}$ and $\mathrm{H}_{2} \mathrm{O}$ both show a large increase in the first $3 \times 10^{5} \mathrm{yr}$ due to the large influx of icy pebbles. At later times, the abundances are decreased again as the volatiles are accreted onto the star. After $3 \times 10^{6} \mathrm{yr}$ the $\mathrm{H}_{2} \mathrm{O}$ and $\mathrm{CO}_{2}$ abundances are lower than $10^{-5}$.

\subsection{Viscous evolution and $\mathrm{CO}_{2}$ destruction}

The models shown in Figs. 3-5 without any chemical processing predict $\mathrm{CO}_{2}$ abundances that are very high in the inner regions, $10^{-5}-10^{-4}$, orders of magnitude higher that the value of $\sim 10^{-8}$ inferred from observations (Pontoppidan \& Blevins 2014; Bosman et al. 2017). There are multiple explanations for this disparity, both physical and chemical, which are discussed in Sect. 5. Here we investigate how large any missing chemical destruction route for gaseous $\mathrm{CO}_{2}$ would need to be. As discussed in Sect. 3 and Appendix B.1, midplane $\mathrm{CO}_{2}$ can only be destroyed by cosmic-ray induced processes in the current networks. However, both cosmic ray induced photodissociation and $\mathrm{He}^{+}$production are an order of magnitude slower than the viscous mixing time. We therefore introduce additional destruction of gaseous $\mathrm{CO}_{2}$ with a rate that is a constant over the 

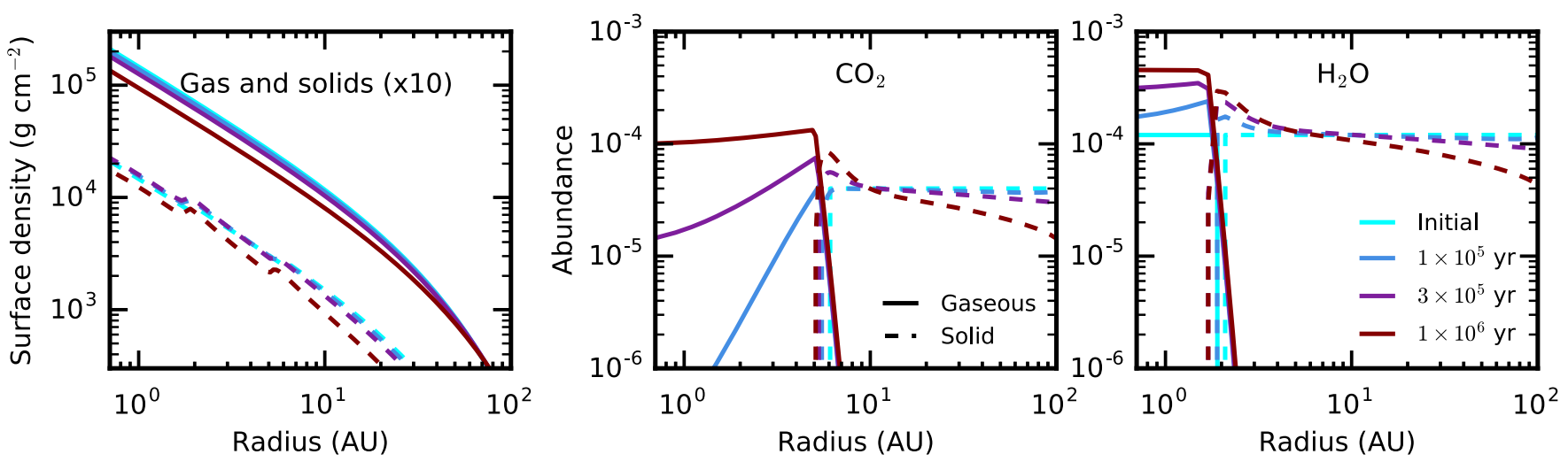

Fig. 4. Time evolution series for a model with grain growth. This model assumes an $\alpha$ of $10^{-3}$. The fragmentation velocity for this model is $3 \mathrm{~m} \mathrm{~s}{ }^{-1}$. Panels as in Fig. 3.
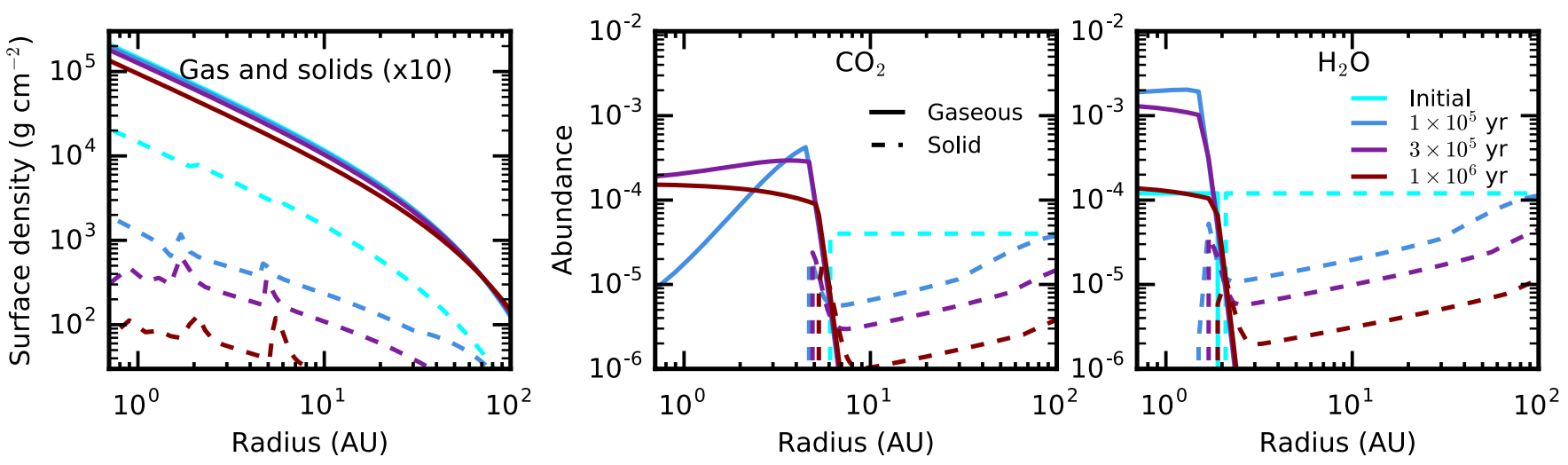

Fig. 5. Time evolution series for a model with grain growth. The fragmentation velocity for this model is $30 \mathrm{~m} \mathrm{~s}^{-1}$ and $\alpha=10^{-3}$. The vertical scales are different from Figs. 3 and 4. Panels as in Fig. 3.

entire disk. This rate is varied between $10^{-13}$ and $10^{-9} \mathrm{~s}^{-1}$ to obtain agreement with observations. For comparison, the cosmic ray induced process generally have a rate of the order of $10^{-14} \mathrm{~s}^{-1}$ (see Sect. 3.2.2). The rate is implemented as an effective destruction rate, so there is no route back to $\mathrm{CO}_{2}$ after destruction. To get the absolute rate one has to also take into account the reformation efficiency, but that can be strongly dependent on the destruction pathway, of which we are agnostic. These efficiencies are discussed in Sect. 5 and Appendix B.

Figure 6 shows the abundance evolution for a model where only gaseous $\mathrm{CO}_{2}$ is destroyed, at a rate of $10^{-11} \mathrm{~s}^{-1}$. In this case the innermost parts of the disk are empty of $\mathrm{CO}_{2}$, whereas the abundance of $\mathrm{CO}_{2}$ reaches values close to the initial ice abundances around the iceline. Due to the constant destruction of $\mathrm{CO}_{2}$ near the iceline, the actual ice abundance of $\mathrm{CO}_{2}$ is also lower than the initial value.

The left panel of Fig. 7 shows the $\mathrm{CO}_{2}$ abundance distribution for models with $\alpha=10^{-3}$. The abundance profiles after $1 \mathrm{Myr}$ of evolution are presented, when a semi-steady state has been reached. The peak abundance and peak width of the $\mathrm{CO}_{2}$ gas abundance profile both depend on the assumed destruction rate: a higher rate leads to a lower peak abundance and a narrower peak, while a lower rate leads to the opposite. A rate of $\sim 10^{-11} \mathrm{~s}^{-1}$ or higher is needed to decrease the average gaseous $\mathrm{CO}_{2}$ abundance below the observational limit (see below). Increasing the rate moves the location of the iceline further out, as the total available $\mathrm{CO}_{2}$ near the iceline decreases. The viscosity also influences the width of the abundance profile, higher viscosities lead to a broader abundance peak.

\subsection{Viscous evolution, grain growth and $\mathrm{CO}_{2}$ destruction}

The next step is to include $\mathrm{CO}_{2}$ destruction in viscous evolution models with grain growth. Here only models that retain the disk dust mass, that is, models that have an overall gas-to-dust ratio smaller than 1000 after $1 \mathrm{Myr}$ of evolution are considered since there is no observational evidence for very high gas-todust ratios. This means that the grains in our models do not reach pebble sizes such as have been inferred from observations (e.g. Pérez et al. 2012; Tazzari et al. 2016). It is unclear how these large grains are retained in the disk as the radial drift timescale for these particles is expected to be shorter than the disk lifetime (e.g. Birnstiel et al. 2010; Krijt et al. 2016). The requirement of dust retention restricts our models to $\alpha=10^{-4}$ and $u_{f}=1 \mathrm{~m} \mathrm{~s}^{-1}, \alpha=10^{-3}$ and $u_{f}=1,3 \mathrm{~m} \mathrm{~s}^{-1}$, and $\alpha=10^{-2}$ and $u_{f}=1,3,10 \mathrm{~m} \mathrm{~s}^{-1}$.

The abundance profiles for different $\mathrm{CO}_{2}$ destruction rates are shown in the right panel of Fig. 7 for the model with $\alpha=10^{-3}$ and $u_{f}=3 \mathrm{~m} \mathrm{~s}^{-1}$. The abundance profiles are determined after $1 \mathrm{Myr}$, when the disk is in a semi-steady state. A destruction rate higher than $10^{-12} \mathrm{~s}^{-1}$ will create an abundance profile with clear abundance maximum at the iceline, but for this rate $10^{-12} \mathrm{~s}^{-1}$ the disk surface area averaged $\mathrm{CO}_{2}$ abundance is still higher than the observed value for both cases. Only for a rate of around $10^{-10} \mathrm{~s}^{-1}$ do the models predict an abundance profile with a disk surface area averaged $\mathrm{CO}_{2}$ abundance that is consistent with the high end of the observations. Only the models with destruction rate higher than $10^{-9} \mathrm{~s}^{-1}$ have a peak in the abundance that is consistent with the maximal inferred abundances from the observations. As shown in Fig. 7 the model with a rate of $10^{-11} \mathrm{~s}^{-1}$ will create a 
A. D. Bosman et al.: Efficiency of radial transport of ices: the case of $\mathrm{CO}_{2}$
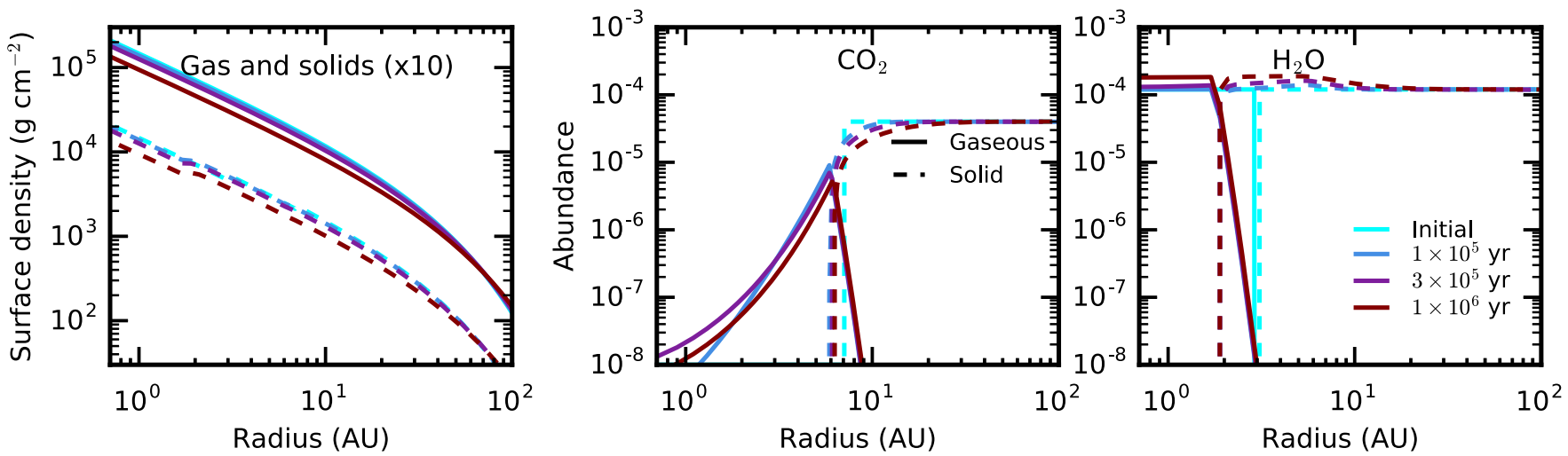

Fig. 6. Time evolution series for a model without grain growth but with a constant destruction rate of gaseous $\mathrm{CO}_{2}$ of $10^{-11} \mathrm{~s}^{-1}$. This model assumes an $\alpha$ of $10^{-3}$. Panels as in Fig. 3, but with different vertical scales.

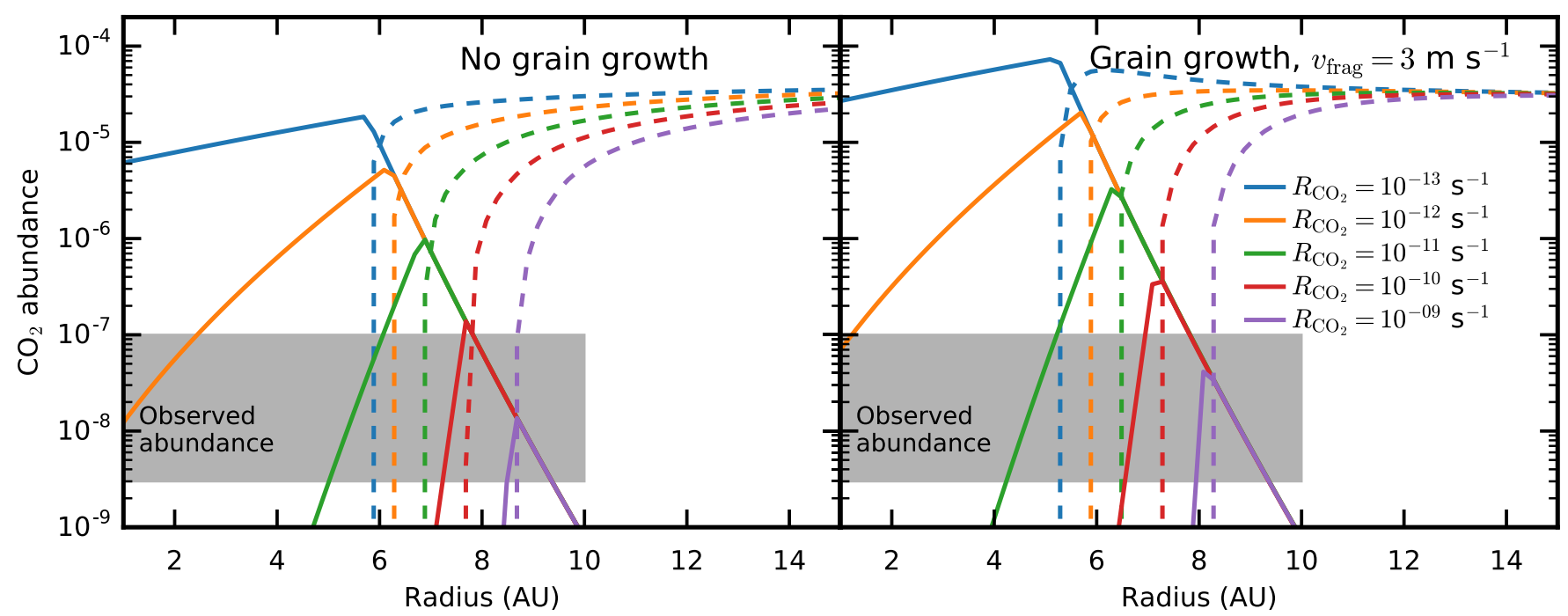

Fig. 7. Abundance of $\mathrm{CO}_{2}$ as function of radii for models with different destruction rates of $\mathrm{CO}_{2}$. These model use $\alpha=10^{-3}$ and $u_{f}=0 \mathrm{~m} \mathrm{~s}{ }^{-1}(l e f t)$ and $u_{f}=3 \mathrm{~m} \mathrm{~s}^{-1}$ (right). Abundance profiles after $1 \mathrm{Myr}$ of evolution are shown. At this point the disk has reached a semi steady state. A destruction rate of at least $10^{-11} \mathrm{~s}^{-1}$ is needed to keep the averaged $\mathrm{CO}_{2}$ abundance below the observational limit. The iceline is further out for models with higher destruction rates as the destruction of gas-phase $\mathrm{CO}_{2}$ lowers the total abundance of $\mathrm{CO}_{2}$ within $10 \mathrm{AU}$ where $\mathrm{CO}_{2}$ can sublimate.

spectrum consistent with the observations, even though the average $\mathrm{CO}_{2}$ abundance is higher than the observed abundance limit.

For the selected models, the effect of different fragmentation velocities is small, with differences in peak abundances less than a factor of five between similar models, as seen in Fig. 7. This is unsurprising as the selection criteria for the models only consider models that have a transport velocity of solids that is less than an order of magnitude faster than the transport velocity of the model without grain growth and radial drift. For models with faster growth it becomes more arbitrary to give a representative abundance profile as semi steady state is not reached at any point in time before the disk is depleted of most of the dust and volatiles in the inner regions. However, for very young disks that may have very efficient radial drift of grains, a high gas-phase $\mathrm{CO}_{2}$ abundance is expected within the $\mathrm{CO}_{2}$ iceline unless the destruction rate is $10^{-11} \mathrm{~s}^{-1}$ or higher.

\subsection{Model spectra}

The spectral modelling the focuses on the $\mathrm{CO}_{2}$ bending mode around centred at $15 \mu \mathrm{m}$. This bending mode has a strong $Q$ branch that has been observed by Spitzer in protoplanetary disks
(Carr \& Najita 2008; Pontoppidan et al. 2010). This region also has a weaker feature due to the $Q$-branch of ${ }^{13} \mathrm{CO}_{2}$ at $15.42 \mu \mathrm{m}$ which can be used, together with the ${ }^{12} \mathrm{CO}_{2} Q$-branch, to infer information on the abundance structure (Bosman et al. 2017).

For the model with $\alpha=10^{-3}$ and $R_{\text {destr, } \mathrm{CO}_{2}}=10^{-11}$, the 2D abundance distribution assumed for the ray-tracing is shown in Fig. 8. The enhanced $\mathrm{CO}_{2}$ abundance around the iceline shows up clearly. The results of the continuum ray-tracing can be found in Fig. 5 of Bosman et al. (2017). The temperature at $1 \mathrm{AU}$ is $320 \mathrm{~K}$. This is higher than the value assumed for the models in the previous sections and is in line with the high luminosity of the modelled source of $7.7 L_{\odot}$. The higher temperature moves the $\mathrm{CO}_{2}$ iceline slightly further out. The abundance profiles from the viscous model with dust growth and $\mathrm{CO}_{2}$ destruction are still very similar to those from the previous section.

Figure 8 also shows the emitting region of the $\mathrm{CO}_{2} Q(6)$ line for both isotopologues. This is one of the strongest lines in the $\mathrm{CO}_{2}$ spectrum and part of the $Q$-branch. As such it is a good representative for the complete $Q$-branch. Due to its high abundance, the ${ }^{12} \mathrm{CO}_{2}$ lines have a high optical depth over a larger area in the disk. As such a large part of the disk contributes to the emission of the ${ }^{12} \mathrm{CO}_{2}$ lines. For ${ }^{13} \mathrm{CO}_{2}$ the emission is from a more compact area, close to the peak in $\mathrm{CO}_{2}$ abundance. 


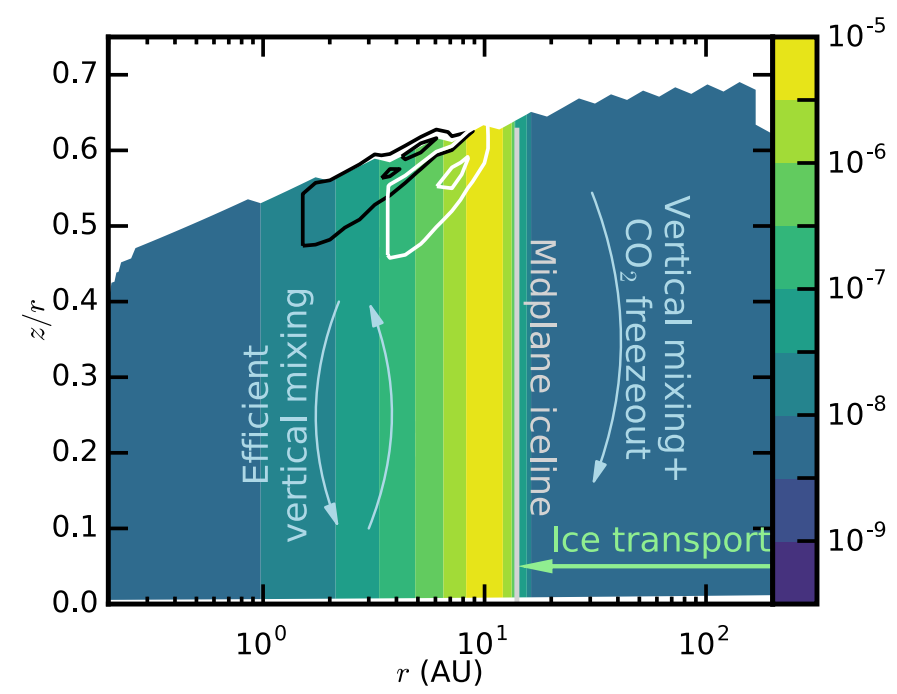

Fig. 8. $2 \mathrm{D} \mathrm{CO}_{2}$ abundance distribution from the $R_{\text {destr, } \mathrm{CO}_{2}}=10^{-11}$ model with an abundance floor of $10^{-9}$. Black and white lines show the regions where $25 \%$ and $75 \%$ of the emission of respectively ${ }^{12} \mathrm{CO}_{2}$ and ${ }^{13} \mathrm{CO}_{2}$ is emitted from. The ${ }^{13} \mathrm{CO}_{2}$ emission is more concentrated around the region with the abundance enhancement.

Figure 9 shows $\mathrm{CO}_{2}$ spectra for different destruction rates and abundance floor of $10^{-8}$. Line-to-continuum ratios for the individual $R$ and $P$ branch lines are all greater than 0.01 and are detectable by JWST (Bosman et al. 2017). Note that only even $J$ levels exist in the vibrational ground state, so $P, Q$ and $R$ branch lines exist only every other $J$ for this band. Both the ${ }^{12} \mathrm{CO}_{2}$ and the ${ }^{13} \mathrm{CO}_{2} Q$-branch fluxes are influenced by the different destruction rates. For a rate of $10^{-12} \mathrm{~s}^{-1}$ the ${ }^{12} \mathrm{CO}_{2} Q$-branch is a factor of two brighter than for the higher rates, which give fluxes very similar in magnitude for the ${ }^{12} \mathrm{CO}_{2} Q$-branch. For ${ }^{13} \mathrm{CO}_{2}$ the model with a destruction rate of $10^{-12} \mathrm{~s}^{-1}$ is a least a factor of five brighter than the models with higher rates of $10^{-11} \mathrm{~s}^{-1}$ and $10^{-10} \mathrm{~s}^{-1}$. Since the latter two ${ }^{1} 2 \mathrm{CO}_{2}$ spectra are indistinguishable observationally, a conservative lower limit to the destruction rate of $10^{-11} \mathrm{~s}^{-1}$ is chosen. For ${ }^{13} \mathrm{CO}_{2}$ however, there is a factor of three difference between the two higher rate models; here the ${ }^{13} \mathrm{CO}_{2} Q$-branch flux is determined by subtracting the contribution from the ${ }^{12} \mathrm{CO}_{2} P(23)$-line assuming it is the same as that of the neighbouring $P(21)$ line.

The flux ratio of the ${ }^{13} \mathrm{CO}_{2} Q$-branch over the ${ }^{12} \mathrm{CO}_{2} Q$ branch is shown in Fig. 10 for a range of $\mathrm{CO}_{2}$ destruction rates. Three regimes can be seen. For ratios larger than $\sim 0.13$ the $\mathrm{CO}_{2}$ released from the ice dominates the abundance profile and spectrum, due to the relatively low destruction rate. The high $\mathrm{CO}_{2}$ column, due to the high abundance around the iceline sets the small flux ratio. At ratios smaller than 0.03 , corresponding to destruction rates larger than $5 \times 10^{-11} \mathrm{~s}^{-1}$, the $\mathrm{CO}_{2}$ released from the ice at the iceline is destroyed so fast that it cannot leave an imprint on the $Q$-branch fluxes. In the region in between, both the $\mathrm{CO}_{2}$ released at the iceline as well as the upper layer $\mathrm{CO}_{2}$ influence the spectra, as such the flux ratio not only depends on the $\mathrm{CO}_{2}$ destruction rate, but also on the $\mathrm{CO}_{2}$ abundance floor used.

Note that Bosman et al. (2017) show that a high ${ }^{13} \mathrm{CO}_{2} Q$ branch over ${ }^{12} \mathrm{CO}_{2} Q$-branch flux ratio may also be an indication of a high abundance in the inner disk. More generally it can be said that a high flux ratio indicates that the $\mathrm{CO}_{2}$ responsible for the emission is highly optically thick. In these cases the width of

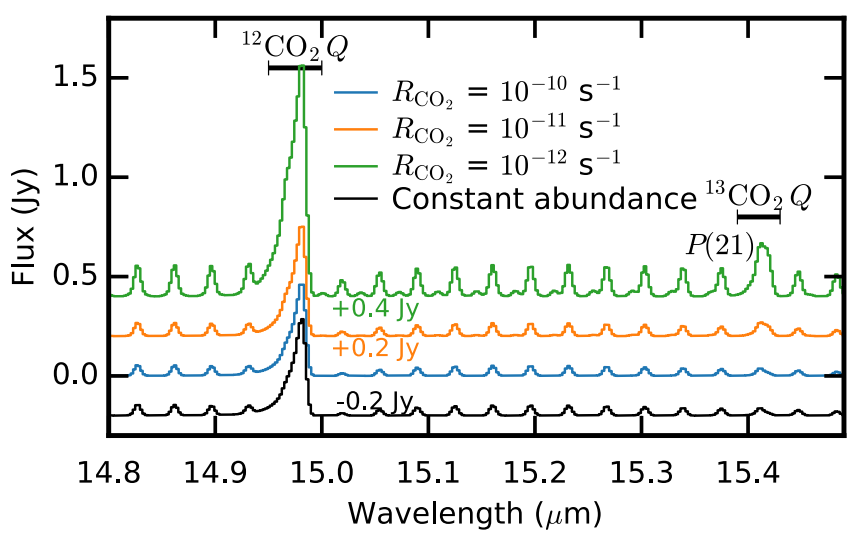

Fig. 9. Spectra ${ }^{12} \mathrm{CO}_{2}$ and ${ }^{13} \mathrm{CO}_{2}$ for models with different destruction rates at a spectral resolving power $R=2200$ appropriate for JWSTMIRI. The abundance floor is taken to be $10^{-8}$. The black line shows the model results for a constant abundance as used in Bosman et al. (2017). The regions were the $Q$-branch flux is extracted is shown in the black lines. The ${ }^{13} \mathrm{CO}_{2} Q$-branch is blended with the ${ }^{12} \mathrm{CO}_{2} P(23)$ line. The neighbouring $P(21)$ line is indicated. Note that the models with destruction rates of $10^{-11}$ and $10^{-10} \mathrm{~s}^{-1}$ are indistinguishable in their ${ }^{12} \mathrm{CO}_{2}$ spectra.

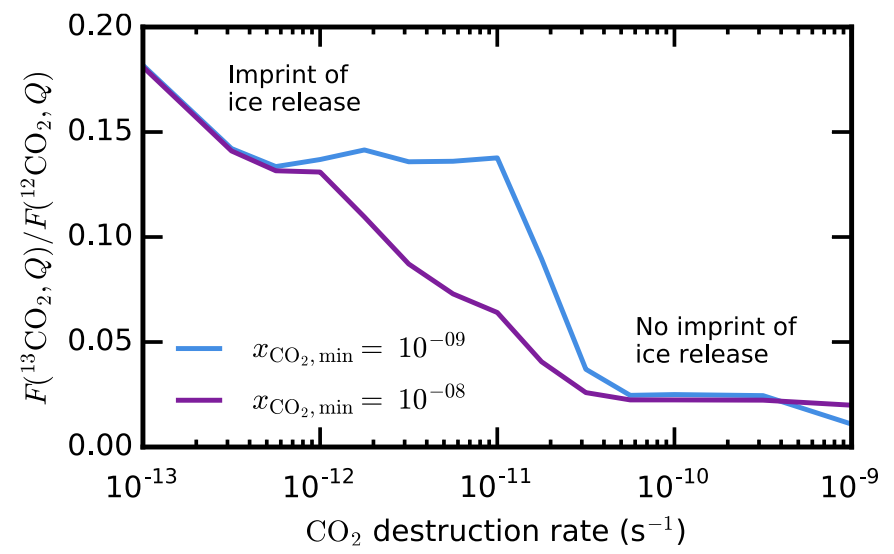

Fig. 10. Flux ratios of the ${ }^{13} \mathrm{CO}_{2}$ and ${ }^{12} \mathrm{CO}_{2} Q$-branches, determined by integrating the region shown in Fig. 9. The ${ }^{13} \mathrm{CO}_{2} Q$-branch flux is corrected for the emission of the $P(23)$ line by subtraction of the neighbouring $P(21)$ line flux.

the ${ }^{13} \mathrm{CO}_{2} Q$-branch can be a measure for the temperature of this emitting gas, which can be related to the radial location of the origin of the emission. An analysis like this will probably only be possible with disk specific modelling.

The spectral modelling done here is meant as an illustration to what could be possible with JWST-MIRI observations and to show what kind of spectral features could hold clues to new insights into disk dynamics. As such the model setup is not entirely self consistent. Foremost, the temperature profiles calculated from the radiative transfer are not exactly the same as the temperature profile used in the viscous disk model. Furthermore, the starting point for our viscous evolution is rather arbitrary.

Another uncertainty is the vertical distribution of the $\mathrm{CO}_{2}$ sublimated from the ices. The assumed turbulent viscosity would not only mix material radially, but also vertically, and the $\mathrm{CO}_{2}$ that comes off the grain near the midplane should make it up to the upper layers of the disk where the infrared emission is generated. 
The vertical dispersion of a species injected into the disk midplane grows with time as $\sigma_{\mathrm{X}}=\sqrt{D_{\text {gas }} t}$ (Ciesla 2010). A steady state is reached if $\sigma_{X}=H$, the scale height of the disk, at which point a constant abundance is reached. As such we can estimate the time needed for vertical mixing. For a self-similar disk, this time scale becomes:

$t_{\text {vert, eq }}=9.1\left(\frac{0.01}{\alpha}\right)\left(\frac{r}{1 \mathrm{AU}}\right)^{0.5+q}\left(\frac{200}{T_{1 \mathrm{AU}}}\right)^{q}\left(\frac{h_{\mathrm{FWHM}}}{0.05}\right) \mathrm{yr}$.

If a similar mixing speed $(\alpha)$ is assumed for the radial and vertical processes, then vertical mixing should happen well within the lifetime of the disk for locations near the $\mathrm{CO}_{2}$ iceline.

Given these uncertainties, a simple constant abundance up to a certain height has been chosen. However higher up in the disk, photodissociation by stellar radiation and processing by stellar X-rays become important. $\mathrm{CO}_{2}$ is removed in our model in the harshest environments $\left(A_{\mathrm{V}}<1 \mathrm{mag}\right)$, but $\mathrm{CO}_{2}$ photodissociation is still possible in the upper disk regions where $\mathrm{CO}_{2}$ is included. The location and thickness of this layer is discussed in Sects. 5.2.5 and 5.2.6.

\section{Discussion}

Viscous disk models including only freeze-out and desorption of $\mathrm{CO}_{2}$ predict a higher $\mathrm{CO}_{2}$ abundance than observed. This conclusion holds even for models without grain growth and radial drift. The discussion is divided into two sections, first the gas and grain surface chemistry is discussed to explore alternative chemical destruction routes. Subsequently physical effects are investigated, some of which also imply chemical effects to happen. Figure 11 shows the $\mathrm{CO}_{2}$ abundance structure in the disk with some of the mechanisms discussed here that could obtain agreement between observations and models.

\subsection{Chemical processes}

In the scenario that the $\mathrm{CO}_{2}$ released at the iceline is destroyed, an effective destruction rate of $10^{-11} \mathrm{~s}^{-1}$ or higher is required to match the observed and computed abundances. In Sects. 3.2.2 and 3.2.3 a number of known destruction processes for midplane $\mathrm{CO}_{2}$ were discussed, but none of these chemical processes is able to account for the destruction rate of $10^{-11} \mathrm{~s}^{-1}$.

\subsubsection{Clues from high-mass protostars?}

Our finding that a $\mathrm{CO}_{2}$ ice desorption model is inconsistent with $\mathrm{CO}_{2}$ gas-phase observations is not the first to do so. For high mass protostars Boonman et al. (2003) noted a similar disagreement between simple in-fall and desorption models, which predict $\mathrm{CO}_{2}$ gas-phase abundances around $10^{-5}$ whereas observations are more consistent with an abundance of $\sim 10^{-7}$, a two orders of magnitude difference. Assuming that the $\mathrm{CO}_{2}$ ice sublimates and is destroyed within the dynamical time of $10^{4} \mathrm{yr}$, a destruction rate of at least $10^{-11} \mathrm{~s}^{-1}$ is needed for protostars as well, of the same order as the rate needed in disks. If the $\mathrm{CO}_{2}$ destruction mechanism is similar in both types of sources, then this would most likely be a chemical destruction mechanism with no or a weak dependence on total density since densities in protostellar envelopes are orders of magnitude lower than in the inner disk midplanes and the physical environments are very different. However, as argued below the existence of such a chemical pathway is unlikely, except for destruction by UV photons or X-rays.

\subsubsection{Alternative gas phase destruction routes}

Here we investigate whether a chemical pathway could be missing in the chemical networks. Since ion-molecule reactions initiated by $\mathrm{He}^{+}$have already been discarded, the assumption is that this destruction comes from a neutral-neutral reactive collision. The rate for a two-body reaction can be written as:

$R_{\mathrm{CO}_{2}, \text { destr }}=f_{X} k_{2 \text {-body }} n_{\text {gas }} x_{X}$

where $n_{\text {gas }}$ is the gas number density, $k_{2 \text {-body }}$ is the two-body reaction rate coefficient, $x_{X}$ is the abundance of the collision partner and $f_{X}$ is the branching ratio to any product that does not cycle back to $\mathrm{CO}_{2}$. Near the iceline densities are around $10^{13} \mathrm{~cm}^{-3}$, a typical rate coefficient for a barrier-less neutralneutral reaction is around $10^{-11} \mathrm{~cm}^{3} \mathrm{~s}^{-1}$. As such the partner abundance needs to be higher than $10^{-13}$ to destroy $\mathrm{CO}_{2}$ at a fast enough rate. The only species that have a nearly barrierless reaction with $\mathrm{CO}_{2}$ in either the UMIST (McElroy et al. 2013) or KIDA (Wakelam et al. 2012) databases are $\mathrm{C}, \mathrm{CH}_{2}$ and $\mathrm{Si}$. Reaction rates for $\mathrm{CO}_{2}$ with $\mathrm{C}$ to form two $\mathrm{CO}$ molecules and with $\mathrm{CH}_{2}$ to form $\mathrm{CO}+\mathrm{H}_{2} \mathrm{CO}$ are $10^{-15} \mathrm{~cm}^{3} \mathrm{~s}^{-1}$ and $3.9 \times$ $10^{-14} \mathrm{~cm}^{3} \mathrm{~s}^{-1}$ (Tsang \& Hampson 1986), however, both rates are high temperature estimates and extrapolated to low temperatures (Hébrard et al. 2009). The rate for $\mathrm{CO}_{2}+\mathrm{Si} \longrightarrow$ $\mathrm{CO}+\mathrm{SiO}$ has been measured at room temperature to be $1.1 \times$ $10^{-11} \mathrm{~cm}^{3} \mathrm{~s}^{-1}$ (Husain \& Norris 1978), McElroy et al. (2013) assume a small energy barrier of $282 \mathrm{~K}$, in line with the high temperature measurements. Other species in the databases that have an exothermic reaction with $\mathrm{CO}_{2}$ are $\mathrm{CH}$ and $\mathrm{N}$; both of these reactions are thought to have a barrier of 3000 and 1710 K respectively (Mitchell 1984; Avramenko \& Krasnen'kov 1967). Other neutral-neutral reactions in the chemical networks are highly endothermic, for example $\mathrm{CO}_{2}+\mathrm{S} \longrightarrow \mathrm{CO}+\mathrm{SO}$ is endothermic by $\sim 20000 \mathrm{~K}$ (Singleton \& Cvetanovic 1988), effectively nullifying this reaction in the temperature range we are interested in.

$\mathrm{C}, \mathrm{CH}_{2}$ and $\mathrm{Si}$ are, due to their high reactivity, only present in very low quantities in the gas-phase with abundances at the solver accuracy limit of $10^{-17}$ for $\mathrm{C}$ and $\mathrm{CH}_{2}$, and $10^{-16}$ for $\mathrm{Si}$ and thus can hardly account for the destruction of a significant amount of $\mathrm{CO}_{2}$ unless it is possible to quickly reform the initial reactant from the reaction products. The reactions products, $\mathrm{CO}, \mathrm{H}_{2} \mathrm{CO}$ and $\mathrm{SiO}$, are again very stable, thus it is unlikely that these molecules quickly react further to form the initial reactants again. This means that the abundance of $\mathrm{C}, \mathrm{CH}_{2}$ and $\mathrm{Si}$ will go down with time if more and more $\mathrm{CO}_{2}$ is added.

\subsubsection{Alternative ice destruction routes}

The low $\mathrm{CO}_{2}$ abundance observed could also be explained if $\mathrm{CO}_{2}$ is converted to other species while in the ice. Bisschop et al. (2007) report that $\mathrm{CO}_{2}$ bombarded with $\mathrm{H}$ does not lead to $\mathrm{HCOOH}$ at detectable levels in their experiments. There are no data available on grain surfaces reactions of $\mathrm{CO}_{2}$ ice with $\mathrm{N}$, $\mathrm{Si}$ or C. If any of these reaction pathways were to destroy $\mathrm{CO}_{2}$ in the ice, they should be very efficient, $\sim 99 \%$ conversion, but at the same time be able to explain the high $\mathrm{CO}_{2}$ ice abundances in comets. This quickly limits the temperature range in which these reactions can be efficient to $30-80 \mathrm{~K}$.

In short, due to the high stability of $\mathrm{CO}_{2}$, only a handful of highly reactive radicals or atoms can destroy $\mathrm{CO}_{2}$, whether in the gas or ice. None of these species is predicted to be abundant enough to quickly destroy the large flux of $\mathrm{CO}_{2}$ that is brought 


\section{A. Disk overview}

- Grain w.o. $\mathrm{CO}_{2}$ ice
- Grain with $\mathrm{CO}_{2}$ ice
B. Gas-phase destruction

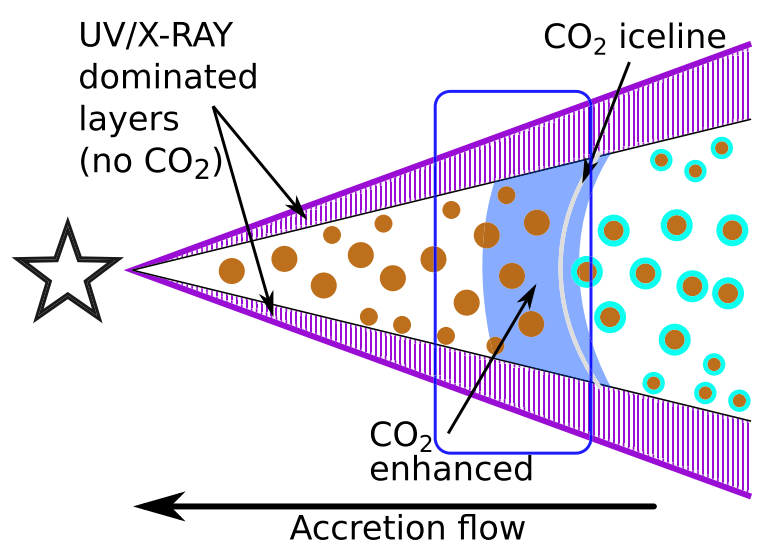

C. Deep UV/X-ray penetration

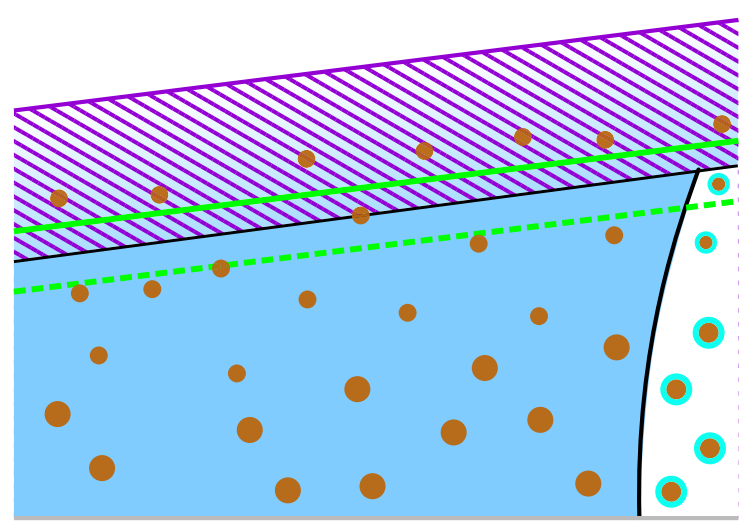

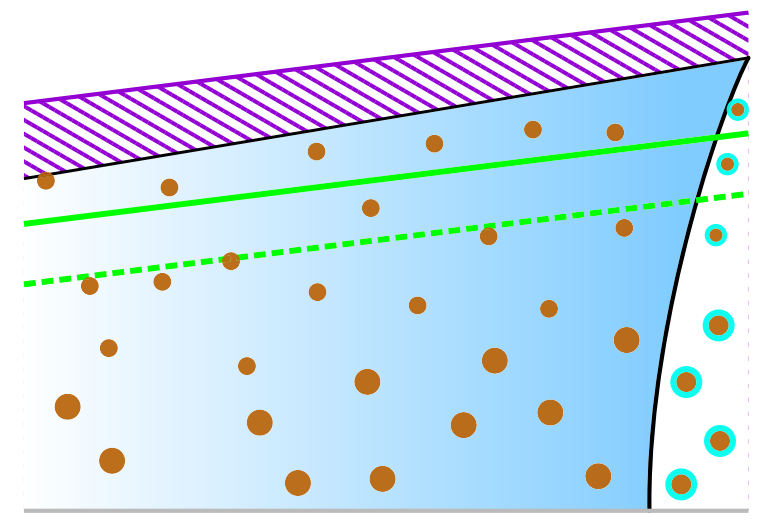

High $\mathrm{CO}_{2}$
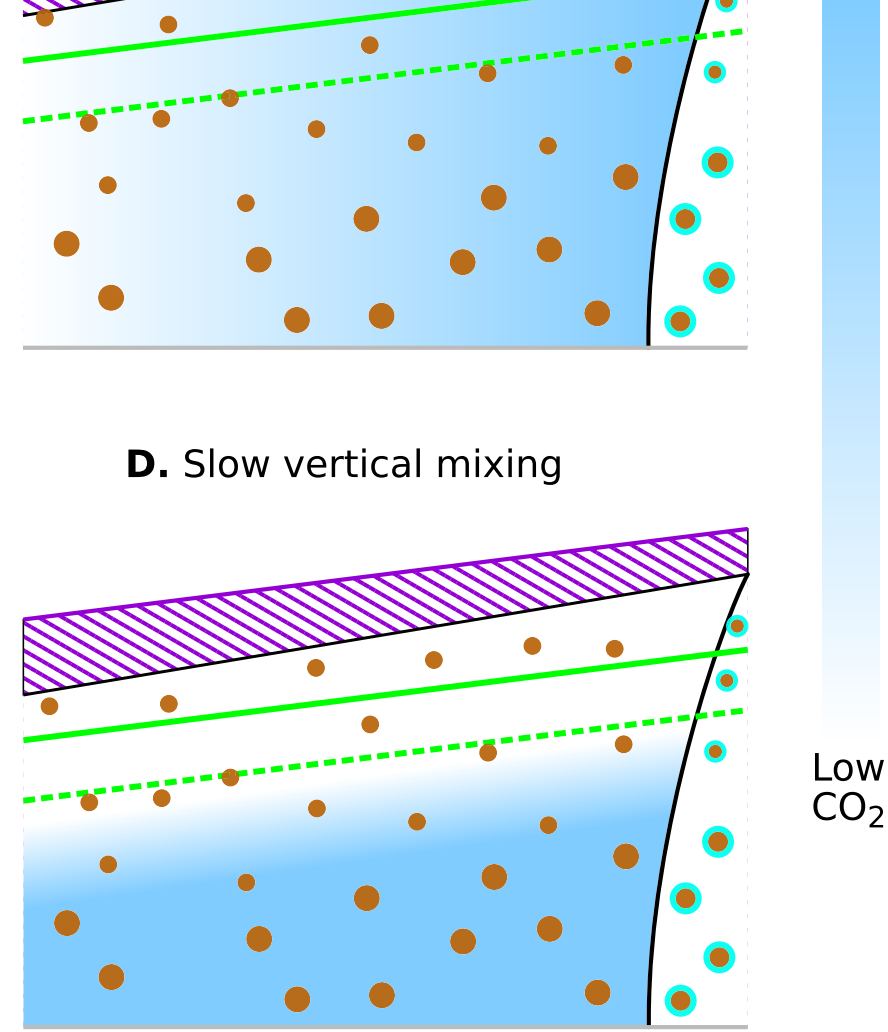

Fig. 11. Schematic representation of the $\mathrm{CO}_{2}$ abundance near the iceline in the $r-z$ plane. Panels $B, C$ and $D$ approximately represent the top half of the blue box in panel $A$. The straight green lines denote the $\tau=1$ surface for ${ }^{12} \mathrm{CO}_{2}$ (solid) and ${ }^{13} \mathrm{CO}_{2}$ (dashed). Panels $B$, $C$ and $D$ : the abundance structure under different mechanism to explain the difference in $\mathrm{CO}_{2}$ abundance between the predictions of the viscous model and the IR observations. Panel B: the case for a constant gas-phase destruction rate due to some as yet unidentified process (Sect. 4.4). Panel $C$ : the abundance structure for deep UV/X-ray penetration (Sects. 5.2 .5 and 5.2.6). In more extreme cases, the ${ }^{13} \mathrm{CO}_{2} \tau=1$ surface can also be in the UV/X-ray dominated region. Panel D: the abundance structure for negligible vertical mixing (Sect. 5.2.1). Image not to scale.

into the inner disk due to dynamic processes of $\mathrm{CO}_{2}$-containing icy grains.

\subsection{Physical processes}

The abundance profiles computed in this work all assume that the $\mathrm{CO}_{2}$ can move freely through the disk, both in radial and vertical directions, while being shielded from UV and $\mathrm{X}$-ray radiation. It is also assumed that all $\mathrm{CO}_{2}$ ice sublimates at the $\mathrm{CO}_{2}$ iceline and that $\mathrm{CO}_{2}$ is highly abundant in the ice, as found in the ISM. Several physical processes could make these assumptions invalid. An important constraint is that due to the difference between the ice abundances (around $10^{-5}$ ) and the inferred gas abundance $\left(<10^{-7}\right)$ any process needs reduce the $\mathrm{CO}_{2}$ abundance in the infrared emitting layers by at least two orders of magnitude.

\subsubsection{Impact of reduced turbulence}

The current models assume a standard viscously spreading and evolving disk with a constant $\alpha$ value. Magnetohydrodynamical (MHD) simulations currently favour disks with strongly changing viscosity in radial and vertical directions (e.g. Gammie 1996; Turner et al. 2007; Bai \& Stone 2013). In these simulations turbulence is only expected to be high at those locations where the ionisation fraction of the gas is also high, that is, the upper and outer regions of the disk. In the disk mid-plane the viscous $\alpha$ may be as low as $10^{-6}$, especially if non-ideal MHD effects are taken into account. In this regime, radial and vertical mixing due to the turbulence becomes a very slow process, with mixing time-scales close to the disk lifetime. Laminar accretion through the midplane would still enrich the inner disk midplane with $\mathrm{CO}_{2}$ but the low vertical mixing would greatly reduce the amount of material that is lifted into the IR-emitting layers of the disk. A mixing time of $10^{6} \mathrm{yr}$ would imply an effective vertical $\alpha$ of $10^{-6}$ for a $\mathrm{CO}_{2}$ iceline at $10 \mathrm{AU}$ (Eq. (36), Ciesla 2010). Note that this still means that $\mathrm{CO}_{2}$ is mixed upwards within $10^{5} \mathrm{yr}$ in the inner $1 \mathrm{AU}$, which would be visible as an added warmer component to the $\mathrm{CO}_{2}$ emission. Observations of another species created primarily on grains at high abundance, $>10^{-7}$, such as $\mathrm{CH}_{4}, \mathrm{NH}_{3}$ or $\mathrm{CH}_{3} \mathrm{OH}$ near their respective icelines can be used to rule out inefficient vertical mixing. 


\subsubsection{Trapping of $\mathrm{CO}_{2}$ in large solids}

There are ways to trap a volatile in a less volatile substance and prevent or delay its sublimation to higher temperatures. One possibility is to lock up the $\mathrm{CO}_{2}$ inside the water ice. This can be done in the bulk of the amorphous ice, below the surface layers, or by the formation of clathrates in the water ice at the high midplane pressures. Even in the case that $\mathrm{CO}_{2}$ is completely trapped in $\mathrm{H}_{2} \mathrm{O}$ ice, $\mathrm{CO}_{2}$ still comes off the grain together with the $\mathrm{H}_{2} \mathrm{O}$ at the $\mathrm{H}_{2} \mathrm{O}$ iceline. At this point the $\mathrm{CO}_{2}$ is in the gas phase and free to diffuse around in the disk. The strong negative abundance gradient outside the $\mathrm{H}_{2} \mathrm{O}$ iceline will transport the $\mathrm{CO}_{2}$ outwards until the $\mathrm{CO}_{2}$ starts to freeze-out of its own accord at the $\mathrm{CO}_{2}$ iceline. This would still result in gas-phase abundances within the $\mathrm{CO}_{2}$ iceline close to the original icy $\mathrm{CO}_{2}$ abundances of the outer disk.

Spherical grains between $0.1 \mu \mathrm{m}$ and $1 \mathrm{~mm}$ are coated with $10^{2}$ to $10^{10}$ layers of water ice, respectively. In the case of small particles, with only 100 layers of ice, it is hard to imagine that the water traps a significant amount of $\mathrm{CO}_{2}$. For the larger grains with a smaller relative surface area, however, the water ice could readily trap a lot of $\mathrm{CO}_{2}$. In a fully mixed $\mathrm{H}_{2} \mathrm{O}$ and $\mathrm{CO}_{2}$ ice, the sublimation of $\mathrm{CO}_{2}$ from the upper 100 million ice layers will still keep the fraction of sublimated $\mathrm{CO}_{2}$ below $1 \%$, however efficient grain fragmentation, such as assumed in our models, will expose $\mathrm{CO}_{2}$ rich layers allowing for the sublimation of more $\mathrm{CO}_{2}$. For small grains, clathrates may keep the $\mathrm{CO}_{2}$ locked-up in the upper layers to prevent sublimation. However, to trap a single $\mathrm{CO}_{2}$ molecule at least six $\mathrm{H}_{2} \mathrm{O}$ molecules are needed (Fleyfel \& Devlin 1991).

For amorphous ices similar restrictions exists on the $\mathrm{H}_{2} \mathrm{O}$ to $\mathrm{CO}_{2}$ ratio for trapping $\mathrm{CO}_{2}$ in the water ice. For ices with a $\mathrm{H}_{2} \mathrm{O}$ to $\mathrm{CO}_{2}$ ratio of $5: 1, \mathrm{CO}_{2}$ desorption happens at the $\mathrm{CO}_{2}$ desorption temperature, only at a ratio of $20: 1$ is the $\mathrm{CO}_{2}$ fully trapped within the $\mathrm{H}_{2} \mathrm{O}$ ice (Sandford \& Allamandola 1990). Collings et al. (2004) show that the majority of the trapped $\mathrm{CO}_{2}$ desorbs at temperatures just below the desorption temperature of $\mathrm{H}_{2} \mathrm{O}$, due to the crystallization of water. Fayolle et al. (2011) note that the fraction of trapped $\mathrm{CO}_{2}$ also depends on the thickness of the ice. They are able to trap $65 \%$ of the $\mathrm{CO}_{2}$ in a $5: 1$ ice, which means that a significant fraction of the $\mathrm{CO}_{2}$ still comes of at the $\mathrm{CO}_{2}$ iceline.

Both of these mechanisms need to have all the $\mathrm{CO}_{2}$ mixed with water. For interstellar dust grains there is observational evidence that this is not the case: high quality Spitzer spectra indicate that $\mathrm{CO}_{2}$ is mixed in both the CO-rich layers of the ice as well as the $\mathrm{H}_{2} \mathrm{O}$-rich layers of the ice (Pontoppidan et al. 2008). Sublimation of $\mathrm{CO}$ from these mixed ices would create a $\mathrm{CO}_{2}$ rich layer around the mixed $\mathrm{H}_{2} \mathrm{O}: \mathrm{CO}_{2}$ inner layers.

Another option would be to lock the $\mathrm{CO}_{2}$ in the refractory material. We note however that the $\mathrm{CO}_{2}$ ice abundance is of the same order as the $\mathrm{Mg}, \mathrm{Fe}$ and $\mathrm{Si}$ abundances (Asplund et al. 2009), as such it is unlikely that $\mathrm{CO}_{2}$ can be locked up in the refractory material without chemical altering that material, such as conversion into, for example, $\mathrm{CaCO}_{3}$.

Measurements from 67P/Churyumov-Gerasimenko indicate that even a kilometre-sized object outgasses $\mathrm{CO}_{2}$ at 3.5 AU distance (Hässig et al. 2015). For larger objects, $\mathrm{CO}_{2}$ retention might be possible, but locking up $99 \%$ of the $\mathrm{CO}_{2}$ ice in these bodies seems highly unlikely.

\subsubsection{Trapping icy grains outside of the iceline}

It is possible to stop the $\mathrm{CO}_{2}$ from sublimating if the $\mathrm{CO}_{2}$ ice never reaches the $\mathrm{CO}_{2}$ iceline, for example by introducing a dust trap (van der Marel et al. 2013). Stopping the $\mathrm{CO}_{2}$ ice from crossing the iceline would also mean stopping the small dust from crossing the iceline. This would lead to fast depletion of the dust in the inner disk, creating a transition disk, although some small dust grains can still cross the gap (Pinilla et al. 2016). Indeed, most disks for which $\mathrm{CO}_{2}$ has been measured have strong nearand mid-infrared continuum emission, indicating that the inner dust disks cannot be strongly depleted. Furthermore, measurements by Kama et al. (2015) show that for Herbig Ae stars, the accreting material coming from a full disk has a refractory material content close to the assumed ISM values even though these disks have no detectable infrared $\mathrm{CO}_{2}$ emission with Spitzer (Pontoppidan et al. 2010).

\subsubsection{Shocks}

For high-mass protostars, Charnley \& Kaufman (2000) proposed that $\mathrm{C}$-shocks with speeds above $30 \mathrm{~km} \mathrm{~s}^{-1}$ passing through the region within the $\mathrm{CO}_{2}$ iceline could account for the low observed $\mathrm{CO}_{2}$ abundance. Dense C-shocks can elevate temperatures above $1000 \mathrm{~K}$ and create free atoms such as $\mathrm{H}, \mathrm{N}$ and $\mathrm{C}$, so all of the $\mathrm{CO}_{2}$ in the shocked layer can then be efficiently converted to $\mathrm{H}_{2} \mathrm{O}$ and $\mathrm{CO}$. At the higher densities in disks, lower velocity shocks can reach the same temperature. For example, Stammler \& Dullemond (2014) calculate that a $5 \mathrm{~km} \mathrm{~s}^{-1}$ shock would reach temperatures upwards of $1000 \mathrm{~K}$ in the post shock gas. If shocks with such speeds occur in disks as well this could be an explanation for the low $\mathrm{CO}_{2}$ abundance. However, assuming the shock only destroys gas-phase $\mathrm{CO}_{2}$, not the ice, shocks must happen at least once every $3 \times 10^{3} \mathrm{yr}\left(1 / 10^{-11} \mathrm{~s}^{-1}\right)$ and the shock speeds have to be of the order of the Keplerian velocity.

These nebular shocks have been invoked to explain the ubiquitous chondrules found in meteorites. For these chondrules to form, dense gas needs to be flash heated, so that grains can evaporate and quickly reform as the gas cools (Hewins et al. 2005). Desch \& Connolly (2002) proposed that a slow shock $\left(7 \mathrm{~km} \mathrm{~s}^{-1}\right)$ in the dense midplane can heat up the gas to temperature around $2000 \mathrm{~K}$, enough to start sublimation (see also Stammler \& Dullemond 2014). In the case of efficient cooling, it takes the gas a few minutes to cool, but this would be enough for the $\mathrm{CO}_{2}+\mathrm{H} \longrightarrow \mathrm{CO}+\mathrm{OH}$ reaction to significantly reduce the $\mathrm{CO}_{2}$ abundance. Slower shocks, with low cooling rates would also be able to destroy $\mathrm{CO}_{2}$ efficiently, as long as temperatures of $750 \mathrm{~K}$ are reached (see Fig. B.1). To effectively destroy $\mathrm{CO}_{2}$, these shocks need to affect the entire inner disk at least once every few thousand years. Each shock could then also induce chondrule formation. There is evidence that chondrules went through multiple creation events, but the frequency of these events is currently not constrained (Ruzicka et al. 2008; Desch et al. 2012).

Further out in the disk, shocks can sputter ices, including $\mathrm{CO}_{2}$ ice, from the grains (Charnley \& Kaufman 2000). However, sputtering does not necessarily destroy the $\mathrm{CO}_{2}$. Moreover, full destruction of ices by shocks is unlikely since $\mathrm{CO}_{2}$ needs to survive in the comet formation regions of the disk.

\subsubsection{UV-dominated layers}

The inner disk chemical models by Walsh et al. (2015) show that there is a surface layer in the disk where UV destruction becomes 

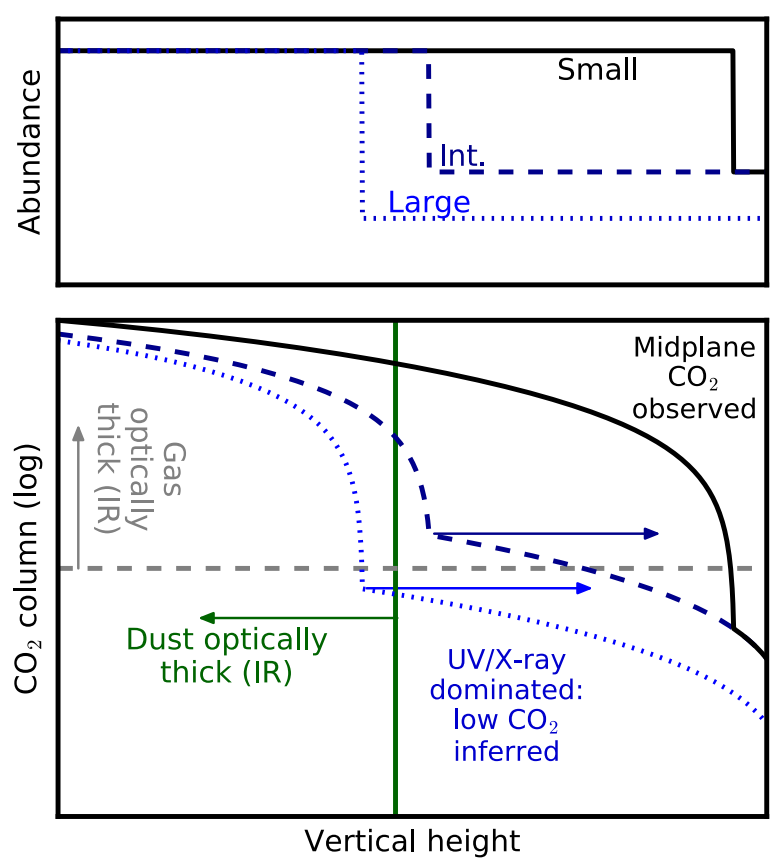

Fig. 12. Schematic of the vertical $\mathrm{CO}_{2}$ abundance (top panel) and upwards column (bottom panel) as function of disk height. Three different cases are shown in solid black, dashed dark blue and dotted blue lines. The black line shows the case for shallow UV or X-ray penetration, in this case an abundance similar to the midplane $\mathrm{CO}_{2}$ abundance will be measured. The dashed dark blue line shows a slightly deeper penetration of radiation, in which case a lower $\mathrm{CO}_{2}$ abundance will be inferred from the observations because the gas is optically thick and hiding the higher abundance deeper into the disk. The dotted blue line shows the case of deep penetration. In this case a low $\mathrm{CO}_{2}$ abundance will be inferred due to the dust hiding the bulk of the $\mathrm{CO}_{2}$. The vertical lines in the upper panel show where the disk becomes optically thick to $\mathrm{CO}_{2}$ lines, the solid and dashed grey line, show where the gas becomes optically thick for the small and intermediate PDR or XDR respectively, the green dotted line shows where the dust becomes optically thick, which is above where the gas becomes optically thick for the large PDR or XDR.

important resulting in abundances of $10^{-8}-10^{-7}$. Depending on the time-scale of the chemical processes and the extent of this layer, it may be optically thick in the ${ }^{12} \mathrm{CO}_{2}$ infrared lines. This means that low abundances of $\mathrm{CO}_{2}$ will be measured in ${ }^{12} \mathrm{CO}_{2}$ irrespective of the amount of sublimating $\mathrm{CO}_{2}$ ice in the midplane. If this UV-dominated layer is not too thick, ${ }^{13} \mathrm{CO}_{2}$ could still have an imprint of the deeper, more abundant $\mathrm{CO}_{2}$ that is coming from the iceline. On the other hand, if this layer is thick enough that even ${ }^{13} \mathrm{CO}_{2}$ becomes optically thick to its own radiation, then the more abundant $\mathrm{CO}_{2}$ sublimating from the iceline would be hidden from our view (see Figs. 10 and 12). This would happen at $\mathrm{CO}_{2}$ columns larger than $10^{18} \mathrm{~cm}^{-2}$.

The (vertical) thickness of the photon dominated layer is very dependent on the UV flux and dust opacity in the upper layers of the disk. For a disk that has only small grains $(<1 \mu \mathrm{m})$ in the upper atmosphere, the UV opacity is high. In this case the UV dominated layer only contains a short column of gas, and thus $\mathrm{CO}_{2}$ (Fig. 13). Observations of such a disk would lead to the spectra as presented in Sect. 4.5. If it is assumed that grains have grown to $\mathrm{mm}$ sizes, even in the upper atmosphere, then the UV can penetrate a lot deeper. This leads to a larger column of UV dominated gas and that could hide the high abundance $\mathrm{CO}_{2}$ in the midplane, if the $\mathrm{CO}_{2}$ in this layer becomes optically thick to its own IR radiation or if the dust becomes optically thick at IR wavelengths in the UV dominated layer. The first scenario is sketched in panel $\mathrm{C}$ of Fig. 11. To probe the midplane $\mathrm{CO}_{2}$ abundance one would need to observe disks with a lot of small grains in the upper atmosphere, preferably around UV weak sources such that the $\mathrm{CO}_{2}$ column in the UV dominated layer is minimised.

\subsubsection{X-ray induced dissociation}

T-Tauri stars are known to have strong X-ray emission $10^{28}$ $10^{34} \mathrm{erg} \mathrm{s}^{-1}$ (Feigelson et al. 2002). These X-rays can ionise the gas and create a local UV field in the same way as cosmic-rays, destroying molecules in the gas (see, for example, the models by Stäuber et al. 2005, for high mass protostars). As X-rays are more quickly attenuated than the higher energy cosmic-rays, they are not efficient in destroying $\mathrm{CO}_{2}$ in the disk midplane, however, X-rays do penetrate further into the disk atmosphere than UV photons. Thus, X-rays can help in decreasing the $\mathrm{CO}_{2}$ abundance in the atmosphere of the disk, especially if hard X-ray flare, such are thought to occur in TW Hya are common in other T-Tauri stars as well (Kastner et al. 2002; Cleeves et al. 2015, 2017).

For our specific DALI model, a total X-ray flux of $10^{30} \mathrm{erg} \mathrm{s}^{-1}$, emitted as a $10^{7} \mathrm{~K}$ black body, would have a destruction rate of $10^{-11} \mathrm{~s}^{-1}$ in most of the ${ }^{12} \mathrm{CO}_{2}$ emitting region and could thus have an effect on the ${ }^{12} \mathrm{CO}_{2}$ fluxes (Fig. 13). A total $\mathrm{X}$-ray flux of $10^{32} \mathrm{erg} \mathrm{s}^{-1}$ would be needed to have the same destruction rate in most of the ${ }^{13} \mathrm{CO}_{2}$ emitting region. Thus, a source that has a time-averaged X-ray luminosity $>10^{30} \mathrm{erg} \mathrm{s}^{-1}$ could show a low $\mathrm{CO}_{2}$ abundance in the atmosphere and have a higher abundance underneath. If the X-ray luminosity of the source is lower than $10^{32} \mathrm{erg} \mathrm{s}^{-1}$, this should be reflected in the ${ }^{13} \mathrm{CO}_{2}$ measurement. Note however that the X-rays do not reach into the midplane mass reservoir. Since the vertical transport for a fully viscous disk is orders of magnitude faster than the radial transport, the replenishment rate of $\mathrm{CO}_{2}$ in the upper layers due to mixing from the midplane would also be faster. Thus, these $\mathrm{X}$-ray luminosities should be considered as lower limits. Most disk hosting stars do not have high enough X-ray luminosities to destroy $\mathrm{CO}_{2}$ efficiently (Bustamante et al. 2016), but X-ray destruction of $\mathrm{CO}_{2}$ in the surface layers can be an explanation in some sources and lead to similar effects as for the case of UV, such as the $\mathrm{CO}_{2}$ IR lines becoming optically thick in the X-ray dominated region.

\section{Summary and conclusions}

We have presented a model for the transport of major gas and ice species. This model predicts that transport of ices from the outer disk towards the inner disk can have a significant effect on the abundance of species in the inner disk. Radial transport is predicted to be so efficient, that even in the case without any radial drift, the modelled $\mathrm{CO}_{2}$ abundance in the inner disk is orders of magnitude higher than the currently available observations of disks. As such $\mathrm{CO}_{2}$ cannot be directly used to trace the mass transport rate. The presence of dust in disks older than a Myr does imply that the mass transport rate of dust cannot be larger than $10 \times \dot{M}_{\text {acc,gas }} /(\mathrm{g} / \mathrm{d})$. This is inconsistent with the pebble-sized dust observed in disks.

To reconcile model and observation $\mathrm{CO}_{2}$ abundances, either the midplane $\mathrm{CO}_{2}$ needs to be destroyed by some physical or chemical mechanism, or the $\mathrm{CO}_{2}$ abundance in the upper layers 
A. D. Bosman et al.: Efficiency of radial transport of ices: the case of $\mathrm{CO}_{2}$
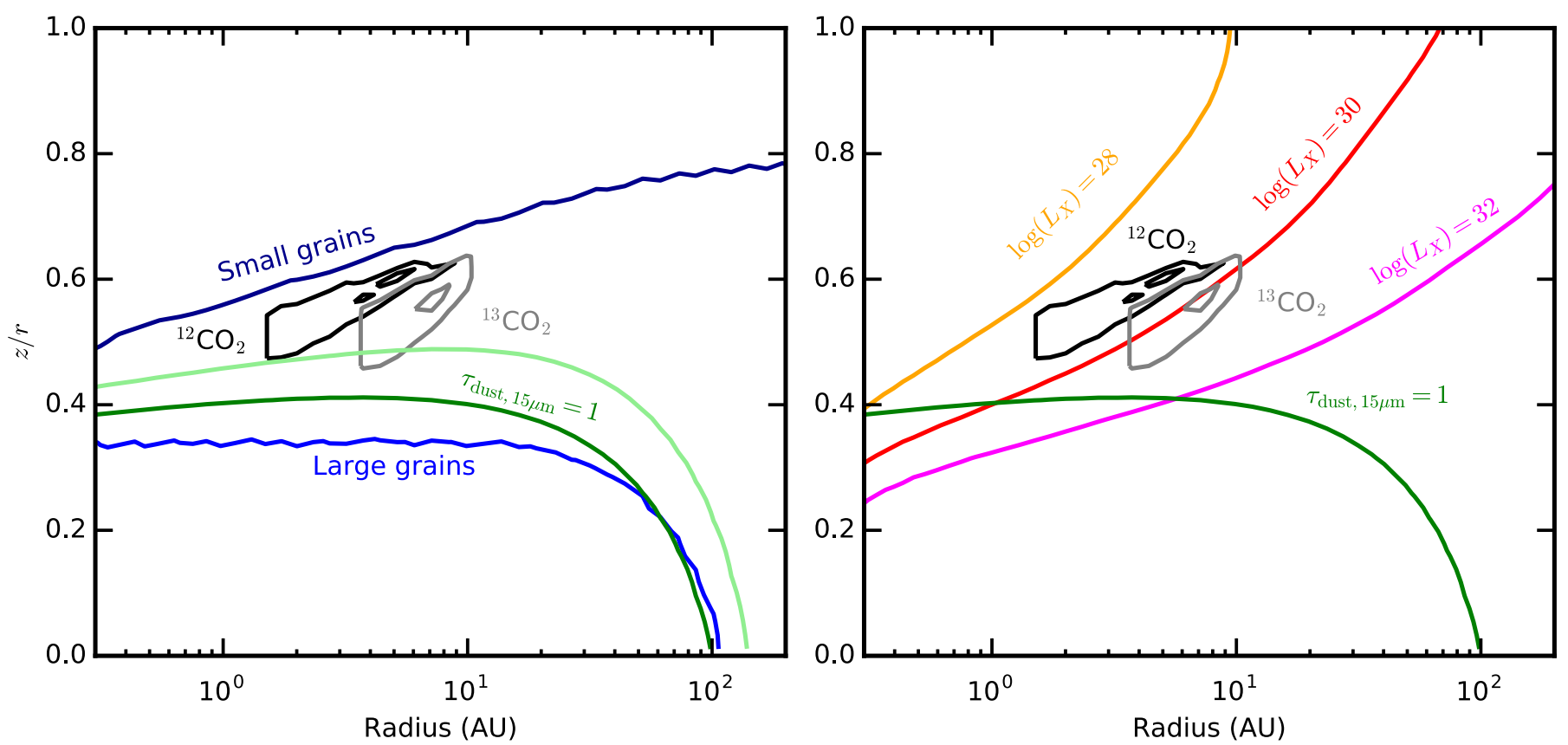

Fig. 13. Left: depth into the disk to which UV can destroy $\mathrm{CO}_{2}$ at a rate of $10^{-11} \mathrm{~s}^{-1}$ for a disk with an upper disk gas-to-dust ratio of 1000 and large grains (cyan) and small grains (blue). The black and grey contours show the area from which $25 \%$ and $75 \%$ of the emission of ${ }^{12} \mathrm{CO}_{2}$ and ${ }^{13} \mathrm{CO}_{2}$ respectively originates. The green lines show the dust $\tau=1$ surface at $15 \mu \mathrm{m}$ for the large grains (up to $1 \mathrm{~mm}$, dark green) and small grains (up to $1 \mu \mathrm{m}$, light green) respectively. The UV photo destruction rate has a stronger dependence on the grain size distribution than this $\mu \mathrm{m}$ dust photosphere. Right: depth into the disk to which X-rays can destroy $\mathrm{CO}_{2}$ at a rate of $10^{-11} \mathrm{~s}^{-1}$ for different stellar X-ray luminosities $\left(L_{X}\right.$ in erg s $\left.{ }^{-1}\right)$. The black and grey contours show the area from which $25 \%$ and $75 \%$ of the emission of ${ }^{12} \mathrm{CO}_{2}$ and ${ }^{13} \mathrm{CO}_{2}$ respectively is coming from. The green line shows the dust $\tau=1$ surface at $15 \mu \mathrm{m}$ for the large grains (up to $1 \mathrm{~mm}$ ).

needs to be decoupled from the $\mathrm{CO}_{2}$ abundance in the midplane. This can be achieved by preventing vertical $\mathrm{CO}_{2}$ cycling, hiding the $\mathrm{CO}_{2}$ under the dust IR photosphere or by the creating a low abundance $\mathrm{CO}_{2}$ column that is optically thick in a UV or X-ray dominated layer. We can summarize this work with the following conclusions.

- Accretion flow and diffusion equilibrate the inner disk $\mathrm{CO}_{2}$ gas abundance with the high outer disk $\mathrm{CO}_{2}$ ice abundance of $\sim 10^{-5}$ in less than $1 \mathrm{Myr}$ for $\alpha>10^{-3}$ (Sect. 4.1).

- For any disk that retains its dust on the same time-scale as it retains the gas, dust diffusion processes dominate over radial drift (Sect. 4.2 and Appendix C).

- If dust can grow efficiently and drift inwards, both $\mathrm{CO}_{2}$ and $\mathrm{H}_{2} \mathrm{O}$ will be enhanced above the outer disk ice abundance as long as the dust is drifting inwards with a mass flux more than $1 \%$ of that of the gas, $\dot{M}_{\text {dust }} \gg 0.01 \dot{M}_{\text {gas }}$ (Sect. 4.4).

- A physical or chemical process that would induce a vertically averaged $\mathrm{CO}_{2}$ destruction rate of at least $10^{-11} \mathrm{~s}^{-1}\left(<10^{3} \mathrm{yr}\right)$ is necessary to produce ${ }^{12} \mathrm{CO}_{2}$ fluxes that are consistent with the current observations of disks (Sect. 4.5).

- Within the current chemical networks, there are no paths to destroy $\mathrm{CO}_{2}$ fast enough without invoking strong $\mathrm{X}$ ray or UV-fluxes or elevated cosmic ray fluxes. Especially at temperatures between 60 and $150 \mathrm{~K}$, destroying $\mathrm{CO}_{2}$ is extremely difficult in the current chemical networks since the formation of $\mathrm{CO}_{2}$ is strongly favoured over the production of $\mathrm{H}_{2} \mathrm{O}$ in that temperature range. Above $300 \mathrm{~K} \mathrm{CO}_{2}$ destruction pathways exists, but they only become efficient enough at temperatures above $750 \mathrm{~K}$ (Sects. 3.2, 5.1 and Appendix B).

- Shocks could raise temperatures enough to destroy $\mathrm{CO}_{2}$ efficiently, but effectively lowering the $\mathrm{CO}_{2}$ abundance would require the entire inner $10 \mathrm{AU}$ of the disk to be processed by a shock every $10^{3} \mathrm{yr}$.

- Trapping $\mathrm{CO}_{2}$ in water ice seems unlikely and is not expected to lower the inner disk gaseous $\mathrm{CO}_{2}$ abundance significantly. Trapping the grains before they cross the iceline efficiently enough to lower $\mathrm{CO}_{2}$ abundances in the inner disk would quickly create a transition disk (Sects. 5.2.2 and 5.2.3).

- Deep penetration of UV and strong X-ray fluxes can lower the $\mathrm{CO}_{2}$ abundance in the ${ }^{12} \mathrm{CO}_{2}$ emitting region. This can happen if grains in the disk atmosphere have grown to $\mathrm{mm}$ sizes and the central source has enough UV luminosity $\left(>10^{27} \mathrm{erg} \mathrm{s}^{-1}\right.$, about $0.1 \%$ of the solar UV). Likewise sources that have an X-ray luminosity greater than $10^{30} \mathrm{erg} \mathrm{s}^{-1}$ efficiently destroy $\mathrm{CO}_{2}$ in a large part of the IR emitting region. If the UV or X-ray dominated region does not reach down to the dust $15 \mu \mathrm{m} \tau=1$ surface, ${ }^{13} \mathrm{CO}_{2}$ can still contain a information on the amount of sublimated $\mathrm{CO}_{2}$. - A high ratio of the ${ }^{13} \mathrm{CO}_{2} Q$-branch flux over the ${ }^{12} \mathrm{CO}_{2}$ $Q$-branch flux $(>0.05)$, can hint at an contribution of sublimating $\mathrm{CO}_{2}$ at the iceline. Sublimating $\mathrm{CO}_{2}$ is, however, not the only way to get high flux ratios. A more complete, source specific analysis would be needed to exclude other possibilities (Sect. 4.5).

In summary, to explain the discrepancy between our models and observations, we either need to invoke frequent shocks in the inner $10 \mathrm{AU}$, or need to hide the abundant midplane $\mathrm{CO}_{2}$ from our view. This can be due to very inefficient mixing or due to deep UV and/or X-ray penetration. In the case of UV penetration, this would imply efficient grain growth and settling, in the case of X-rays, this would be stronger than measured X-ray fluxes for most sources, or frequent X-ray flares. The key to hiding the midplane $\mathrm{CO}_{2}$ is that the low abundance $\mathrm{CO}_{2}$ in the upper layers has 
a high enough column density to become optically thick to its own IR radiation. Trapping the $\mathrm{CO}_{2}$ on the grains, or destroying it in the gas phase near the iceline at ambient temperatures is strongly disfavoured.

Is $\mathrm{CO}_{2}$ a special case in terms of discrepancy between models and observations? Modelling and observations of other molecules abundant in ices, such as $\mathrm{NH}_{3}$ and $\mathrm{CH}_{4}$, may be used to test whether a chemical or physical reason is the origin of the current discrepancy. Ultimately, such data can constrain the mass flow of solids if the chemical composition of the surface layers is sufficiently representative of that in the midplane.

Acknowledgements. Astrochemistry in Leiden is supported by the European Union A-ERC grant 291141 CHEMPLAN, by the Netherlands Research School for Astronomy (NOVA), by a Royal Netherlands Academy of Arts and Sciences (KNAW) professor prize. Daniel Harsono, Stefano Facchini, Kees Dullemond and Til Birnstiel are thanked for insightful discussions and Catherine Walsh and Christian Eistrup for sharing and help with the gas-grain chemical network.

\section{References}

Adams, N. G., \& Smith, D. 1976, J. Phys. B: At. Mol. Phys., 9, 1439 Agúndez, M., Cernicharo, J., \& Goicoechea, J. R. 2008, A\&A, 483, 831 Aikawa, Y., \& Herbst, E. 1999, A\&A, 351, 233

Ali-Dib, M., Mousis, O., Petit, J.-M., \& Lunine, J. I. 2014, ApJ, 785, 125

Andrews, S. M., Wilner, D. J., Hughes, A. M., Qi, C., \& Dullemond, C. P. 2009, ApJ, 700, 1502

Anicich, V. G., Laudenslager, J. B., Huntress, Jr., W. T., \& Futrell, J. H. 1977, J. Chem. Phys., 67, 4340

Ansdell, M., Williams, J. P., van der Marel, N., et al. 2016, ApJ, 828, 46 Asplund, M., Grevesse, N., Sauval, A. J., \& Scott, P. 2009, ARA\&A, 47, 481 Avramenko, L. I., \& Krasnen'kov, V. M. 1967, Bull. Acad. Sci. USSR Div. Chem. Sci. (Engl. Transl.), 16, 501

Bai, X.-N., \& Stone, J. M. 2013, ApJ, 769, 76

Baulch, D. L., Cobos, C. J., Cox, R. A., et al. 1992, J. Phys. Chem. Ref. Data, 21, 411

Bergin, E. A., Cleeves, L. I., Gorti, U., et al. 2013, Nature, 493, 644

Birnstiel, T., Dullemond, C. P., \& Brauer, F. 2010, A\&A, 513, A79

Birnstiel, T., Klahr, H., \& Ercolano, B. 2012, A\&A, 539, A148

Bisschop, S. E., Fuchs, G. W., van Dishoeck, E. F., \& Linnartz, H. 2007, A\&A, 474, 1061

Blum, J., \& Wurm, G. 2008, ARA\&A, 46, 21

Boogert, A. C. A., Gerakines, P. A., \& Whittet, D. C. B. 2015, ARA\&A, 53, 541

Boonman, A. M. S., van Dishoeck, E. F., Lahuis, F., \& Doty, S. D. 2003, A\&A, 399, 1063

Booth, R. A., Clarke, C. J., Madhusudhan, N., \& Ilee, J. D. 2017, MNRAS, 469, 3994

Bosman, A. D., Bruderer, S., \& van Dishoeck, E. F. 2017, A\&A, 601, A36

Brauer, F., Dullemond, C. P., \& Henning, T. 2008, A\&A, 480, 859

Bruderer, S. 2013, A\&A, 559, A46

Bruderer, S., van Dishoeck, E. F., Doty, S. D., \& Herczeg, G. J. 2012, A\&A, 541 A91

Bruderer, S., Harsono, D., \& van Dishoeck, E. F. 2015, A\&A, 575, A94

Bustamante, I., Merín, B., Bouy, H., et al. 2016, A\&A, 587, A81

Carr, J. S., \& Najita, J. R. 2008, Science, 319, 1504

Cazaux, S., \& Tielens, A. G. G. M. 2004, ApJ, 604, 222

Charnley, S. B., \& Kaufman, M. J. 2000, ApJ, 529, L111

Ciesla, F. J. 2010, ApJ, 723, 514

Ciesla, F. J., \& Cuzzi, J. N. 2006, Icarus, 181, 178

Clarke, C. J., \& Pringle, J. E. 1988, MNRAS, 235, 365

Cleeves, L. I., Bergin, E. A., Qi, C., Adams, F. C., \& Öberg, K. I. 2015, ApJ, 799, 204

Cleeves, L. I., Bergin, E. A., Öberg, K. I., et al. 2017, ApJ, 843, L3

Collings, M. P., Anderson, M. A., Chen, R., et al. 2004, MNRAS, 354, 1133

Cridland, A. J., Pudritz, R. E., Birnstiel, T., Cleeves, L. I., \& Bergin, E. A. 2017, MNRAS, 469, 3910

Cyr, K. E., Sears, W. D., \& Lunine, J. I. 1998, Icarus, 135, 537

Debes, J. H., Jang-Condell, H., Weinberger, A. J., Roberge, A., \& Schneider, G. 2013, ApJ, 771, 45

Desch, S. J., \& Connolly, Jr., H. C. 2002, Meteor. Planet. Sci., 37, 183

Desch, S. J., Morris, M. A., Connolly, H. C., \& Boss, A. P. 2012, Meteor. Planet. Sci., 47, 1139

Desch, S. J., Estrada, P. R., Kalyaan, A., \& Cuzzi, J. N. 2017, ApJ, 840, 86
Draine, B. T. 2003, ApJ, 598, 1017

Draine, B. T., \& Lee, H. M. 1984, ApJ, 285, 89

Drozdovskaya, M. N., Walsh, C., van Dishoeck, E. F., et al. 2016, MNRAS, 462, 977

Eistrup, C., Walsh, C., \& van Dishoeck, E. F. 2016, A\&A, 595, A83

Fayolle, E. C., Öberg, K. I., Cuppen, H. M., Visser, R., \& Linnartz, H. 2011, A\&A, 529, A74

Feigelson, E. D., Broos, P., Gaffney, III, J. A., et al. 2002, ApJ, 574, 258

Fleyfel, F., \& Devlin, J. P. 1991, J. Phys. Chem., 95, 3811

Fraser, H. J., Collings, M. P., McCoustra, M. R. S., \& Williams, D. A. 2001, MNRAS, 327, 1165

Gammie, C. F. 1996, ApJ, 457, 355

Gredel, R., Lepp, S., \& Dalgarno, A. 1987, ApJ, 323, L137

Gundlach, B., \& Blum, J. 2015, ApJ, 798, 34

Hartmann, L., Calvet, N., Gullbring, E., \& D’Alessio, P. 1998, ApJ, 495, 385

Hässig, M., Altwegg, K., Balsiger, H., et al. 2015, Science, 347

Heays, A. N., Bosman, A. D., \& van Dishoeck, E. F. 2017, A\&A, 602, A105

Hébrard, E., Dobrijevic, M., Pernot, P., et al. 2009, J. Phys. Chem. A, 113, 11227

Hewins, R. H., Connolly, Jr., H. C., Lofgren, G. E., \& Libourel, G. 2005, in Chondrites and the Protoplanetary Disk, eds. A. N. Krot, E. R. D. Scott, \& B. Reipurth, ASP Conf. Ser., 341, 286

Husain, D., \& Norris, P. E. 1978, Chem. Phys. Lett., 53, 474

Johansen, A., \& Lambrechts, M. 2017, Annu. Rev. Earth Planet. Sci., 45

Kama, M., Folsom, C. P., \& Pinilla, P. 2015, A\&A, 582, L10

Kastner, J. H., Huenemoerder, D. P., Schulz, N. S., Canizares, C. R., \& Weintraub, D. A. 2002, ApJ, 567, 434

Krijt, S., \& Ciesla, F. J. 2016, ApJ, 822, 111

Krijt, S., Ormel, C. W., Dominik, C., \& Tielens, A. G. G. M. 2016, A\&A, 586, A20

Lambrechts, M., \& Johansen, A. 2012, A\&A, 544, A32

Le Roy, L., Altwegg, K., Balsiger, H., et al. 2015, A\&A, 583, A1

Madhusudhan, N., Knutson, H., Fortney, J. J., \& Barman, T. 2014, in Protostars and Planets VI, eds. H. Beuther, R. Klessen, C. Dullemond, \& T. Henning (University of Arizona Press, Tucson), 739

Manara, C. F., Rosotti, G., Testi, L., et al. 2016, A\&A, 591, L3

Markwick, A. J., Ilgner, M., Millar, T. J., \& Henning, T. 2002, A\&A, 385, 632

Mauclaire, G., Derai, R., \& Marx, R. 1978, Int. J. Mass Spectrom. Ion Phys., 26, 289

McClure, M. K., Bergin, E. A., Cleeves, L. I., et al. 2016, ApJ, 831, 167

McElroy, D., Walsh, C., Markwick, A. J., et al. 2013, A\&A, 550, A36

Meijerink, R., Pontoppidan, K. M., Blake, G. A., Poelman, D. R., \& Dullemond C. P. 2009, ApJ, 704, 1471

Miotello, A., van Dishoeck, E. F., Williams, J. P., et al. 2017, A\&A, 599, A113

Mitchell, G. F. 1984, Astrophys. J. Suppl. Ser., 54, 81

Morbidelli, A., \& Raymond, S. N. 2016, J. Geophys. Res., 121, 1962

Noble, J. A., Congiu, E., Dulieu, F., \& Fraser, H. J. 2012, MNRAS, 421, 768

Öberg, K. I., \& Bergin, E. A. 2016, ApJ, 831, L19

Ormel, C. W., \& Cuzzi, J. N. 2007, A\&A, 466, 413

Ormel, C. W., \& Klahr, H. H. 2010, A\&A, 520, A43

Ormel, C., Liu, B., \& Schoonenberg, D. 2017, A\&A, 604, A1

Pérez, L. M., Carpenter, J. M., Chandler, C. J., et al. 2012, ApJ, 760, L17

Pinilla, P., Klarmann, L., Birnstiel, T., et al. 2016, A\&A, 585, A35

Piso, A.-M. A., Öberg, K. I., Birnstiel, T., \& Murray-Clay, R. A. 2015, ApJ, 815, 109

Pontoppidan, K. M., \& Blevins, S. M. 2014, Faraday Discuss., 169, 49

Pontoppidan, K. M., Boogert, A. C. A., Fraser, H. J., et al. 2008, ApJ, 678, 1005

Pontoppidan, K. M., Salyk, C., Blake, G. A., et al. 2010, ApJ, 720, 887

Pontoppidan, K. M., Blake, G. A., \& Smette, A. 2011, ApJ, 733, 84

Prasad, S. S., \& Tarafdar, S. P. 1983, ApJ, 267, 603

Ruzicka, A., Floss, C., \& Hutson, M. 2008, Geochim. Cosmochim. Acta, 72, 5530

Sandford, S. A., \& Allamandola, L. J. 1990, Icarus, 87, 188

Schoonenberg, D., \& Ormel, C. W. 2017, A\&A, 602, A21

Shakura, N. I., \& Sunyaev, R. A. 1973, A\&A, 24, 337

Singleton, D. L., \& Cvetanovic, R. J. 1988, J. Phys. Chem. Ref. Data, 17, 1377

Smith, I. W. M., Herbst, E., \& Chang, Q. 2004, MNRAS, 350, 323

Stammler, S. M., \& Dullemond, C. P. 2014, Icarus, 242, 1

Stammler, S. M., Birnstiel, T., Panić, O., Dullemond, C. P., \& Dominik, C. 2017, A\&A, 600, A140

Stäuber, P., Doty, S. D., van Dishoeck, E. F., \& Benz, A. O. 2005, A\&A, 440, 949

Stevenson, D. J., \& Lunine, J. I. 1988, Icarus, 75, 146

Takeuchi, T., \& Lin, D. N. C. 2002, ApJ, 581, 1344

Talbi, D., \& Herbst, E. 2002, A\&A, 386, 1139

Tan, J. C., Chatterjee, S., Hu, X., Zhu, Z., \& Mohanty, S. 2016, IAU Focus Meeting, 29, 6

Tazzari, M., Testi, L., Ercolano, B., et al. 2016, A\&A, 588, A53 
A. D. Bosman et al.: Efficiency of radial transport of ices: the case of $\mathrm{CO}_{2}$

Testi, L., Birnstiel, T., Ricci, L., et al. 2014, Protostars and Planets VI, eds. H. Beuther, R. Klessen, C. Dullemond, \& T. Henning (University of Arizona Press, Tucson), 339

Trapman, L., Miotello, A., Kama, M., van Dishoeck, E. F., \& Bruderer, S. 2017 A\&A, 605, A69

Tsang, W., \& Hampson, R. F. 1986, J. Phys. Chem. Ref. Data, 15, 1087

Turner, N. J., Sano, T., \& Dziourkevitch, N. 2007, ApJ, 659, 729

Turner, N. J., Fromang, S., Gammie, C., et al. 2014, Protostars and Planets VI, eds. H. Beuther, R. Klessen, C. Dullemond, \& T. Henning (University of Arizona Press, Tucson), 411

Umebayashi, T., \& Nakano, T. 1981, PASJ, 33, 617
Umebayashi, T., \& Nakano, T. 2009, ApJ, 690, 69

van der Marel, N., van Dishoeck, E. F., E. F., Bruderer, S., et al. 2013, Science, 340, 1199

Wakelam, V., Herbst, E., Loison, J.-C., et al. 2012, ApJS, 199, 21

Walsh, C., Millar, T. J., Nomura, H., et al. 2014, A\&A, 563, A33

Walsh, C., Nomura, H., \& van Dishoeck, E. 2015, A\&A, 582, A88

Weidenschilling, S. J. 1977, MNRAS, 180, 57

Weingartner, J. C., \& Draine, B. T. 2001, ApJ, 548, 296

Wolf, S., \& Voshchinnikov, N. V. 2004, Comput. Phys. Commun., 162, 113

Youdin, A. N., \& Lithwick, Y. 2007, Icarus, 192, 588 


\section{Appendix A: UV dust cross sections}

Figure A.1 shows the UV opacities and cross sections for different grain size distributions used in Sect. 3.2.2.

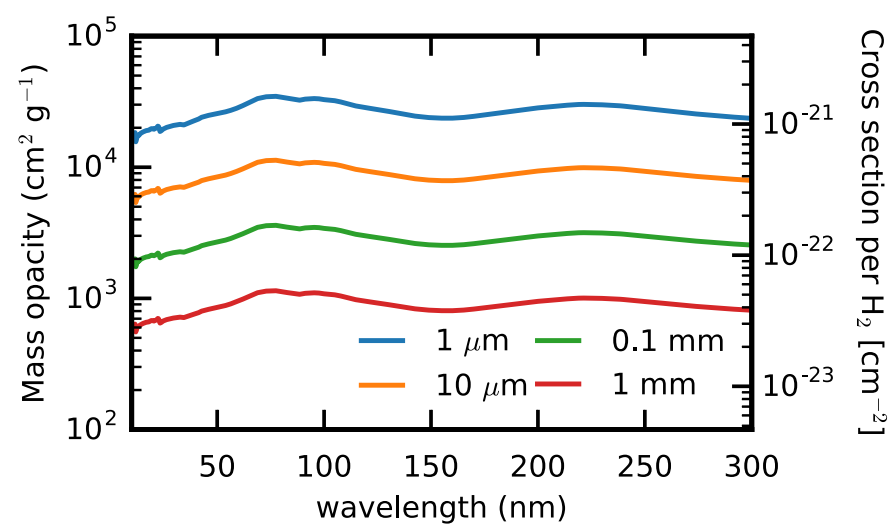

Fig. A.1. UV opacities and cross section of the dust for different sizes of the largest grains. The MRN power law slope is 3.5 for all cases. Growing grains from $1 \mu \mathrm{m}$ to $1 \mathrm{~mm}$ has more than an order of magnitude effect on the opacities.

\section{Appendix B: Chemical modelling}

\section{B.1. Gas-phase only models}

To test the main $\mathrm{CO}_{2}$ destruction and formation processes, a small grid of chemical models was run. The network from Walsh et al. (2014) was used as basis and modified. The gasphase chemical network is based on the UMIST 2012 network (McElroy et al. 2013). The network includes freeze-out and desorption. $\mathrm{H}_{2}$ formation on grains following Cazaux \& Tielens (2004), which includes $\mathrm{H}_{2}$ formation at high temperatures. The grain-surface reactions included in the network were removed for this test. As the main interest is in the midplane layers, the local UV and X-ray flux are assumed to be negligible. The cosmic ray rate is taken to be $10^{-17} \mathrm{~s}^{-1}$.

Four series of models were run, two starting from "atomic" initial conditions (all species are in atomic form except for $\mathrm{H}_{2}$ ), the other two from "molecular" conditions (see Table B.1, the atomic initial conditions use the same elemental abundances as the used for the molecular test). For each set of initial conditions one set of models was run with $\mathrm{H}_{2}$ formation happening only on grains according to Cazaux \& Tielens (2004) leading to an atomic $\mathrm{H}$ abundance of $10^{-4}$ with respect to $\mathrm{H}_{2}$, while in the other set of models the additional $\mathrm{H}_{2}$ formation route at high temperature was added to keep the $\mathrm{H}$ abundance at $10^{-12}$ $\left(1 \mathrm{~cm}^{-3}\right)$. The density was kept constant at $10^{12} \mathrm{~cm}^{-3}$ and the temperature was varied between 150 and $800 \mathrm{~K}$.

The $\mathrm{CO}_{2}$ abundances after $1 \mathrm{Myr}$ of chemical evolution are presented in Fig. B.1. From this plot it can be gathered that there is no efficient destruction mechanism in the current gas-phase network until a temperature of $\sim 450 \mathrm{~K}$ is reached.

For temperatures between 150 and $450 \mathrm{~K}$, the different atomic $\mathrm{H}$ abundances do not significantly influence the results. The difference between the initial $\mathrm{CO}_{2}$ abundance and the $\mathrm{CO}_{2}$ abundance after $1 \mathrm{Myr}$ in the molecular case can be explained
Table B.1. Initial molecular abundances for the chemical models.

\begin{tabular}{llll}
\hline \hline \multicolumn{4}{c}{ Abundances } \\
\hline $\mathrm{H}_{2}$ & $5.0(-1)$ & $\mathrm{H}$ & 0.0 \\
$\mathrm{He}$ & $1.4(-1)$ & $\mathrm{CO}$ & $1.0(-4)$ \\
$\mathrm{CO}_{2}$ & $3.5(-5)$ & $\mathrm{N}_{2}$ & $1.1(-5)$ \\
$\mathrm{N}$ & $2.1(-5)$ & $\mathrm{H}_{2} \mathrm{O}$ & $1.2(-4)$ \\
$\mathrm{Si}$ & $4.0(-10)$ & $\mathrm{H}_{2} \mathrm{~S}$ & $1.9(-9)$ \\
\hline
\end{tabular}

Notes. $a(b)=a \times 10^{b}$.

by the destruction of $\mathrm{CO}_{2}$ and $\mathrm{H}_{2} \mathrm{O}$ due to processes driven by cosmic rays, e.g. cosmic ray induced photo dissociation, and the reformation of $\mathrm{CO}_{2}$ from the fragments.

For models starting from atomic initial conditions the $\mathrm{CO}_{2}$ content decreases with increasing temperature as expected from the formation speeds of $\mathrm{CO}_{2}$ and $\mathrm{H}_{2} \mathrm{O}$ in the model with a low $\mathrm{H}$ abundance, and this trend continues up to higher temperatures. The local minimum in the $\mathrm{CO}_{2}$ abundance at $350 \mathrm{~K}$ is due to reactions with atomic $\mathrm{N}$ before the nitrogen is locked up in other species. When the atomic nitrogen abundance is high, $\mathrm{CO}_{2}$ reacts with $\mathrm{N}$ to form $\mathrm{CO}$ and $\mathrm{NO}, \mathrm{NO}$ is then further processed to $\mathrm{H}_{2} \mathrm{O}$, releasing a $\mathrm{N}$ in the process. This significantly slows down the build-up of $\mathrm{CO}_{2}$ when both $\mathrm{O}$ and $\mathrm{OH}$ are available in the gas-phase in high abundances.

In the models with a high $\mathrm{H}$ abundance, reactions with atomic hydrogen become important starting at $350 \mathrm{~K}$. This process, which is slightly more efficient for $\mathrm{H}_{2} \mathrm{O}$ than for $\mathrm{CO}_{2}$, together with the reformation of $\mathrm{H}_{2} \mathrm{O}$ and $\mathrm{CO}_{2}$ from the resulting $\mathrm{OH}$, sets the equilibrium at high temperature. The abundance of $\mathrm{H}_{2} \mathrm{O}$ as function of temperature is fairly constant, at $10^{-4}$, and thus the $\mathrm{CO}_{2}$ abundance decreases towards the highest temperature, as expected from the formation rate ratio given in Fig. 1. The difference between the $\mathrm{CO}_{2}$ abundance at high temperature in the case of atomic and molecular abundance can be explained by the lower $\mathrm{CO}$ abundance in the case of atomic initial conditions.

For the models with a low $\mathrm{H}$ abundance, $\mathrm{CO}_{2}$ is not efficiently destroyed by $\mathrm{H}$ at high temperatures so the $\mathrm{CO}_{2}$ abundance is nearly constant as function of temperature. From this parameter exploration we can conclude that $\mathrm{CO}_{2}$ can only be effectively destroyed by radicals like $\mathrm{H}$ and $\mathrm{N}$ and that this is only efficient if these are available at high abundances $>10^{-6}$. For the model beginning with atomic abundances the low temperature dependence of the abundance is similar to the high $\mathrm{H}$ abundance case. Above temperatures of $400 \mathrm{~K}$ differences start to be noticeable. The $\mathrm{CO}_{2}$ abundance does not increase with temperature as it does in the case of high $\mathrm{H}$ abundance. This is due to a similar process as in the high $\mathrm{H}$ model around $350 \mathrm{~K}$. All the $\mathrm{CO}_{2}$ that is formed when oxygen is not locked up in water is quickly destroyed by atomic $\mathrm{H}$ and $\mathrm{N}$, when atomic $\mathrm{H}$ and $\mathrm{N}$ have settled to low enough abundances that $\mathrm{CO}_{2}$ can survive, all the $\mathrm{O}$ is already locked up in other species, mostly $\mathrm{H}_{2} \mathrm{O}$ and $\mathrm{CO}$ leaving no extra $\mathrm{O}$ to form $\mathrm{CO}_{2}$. The small amount of $\mathrm{CO}_{2}$ that is in the gas-phase is mostly created by cosmic ray induced destruction of $\mathrm{H}_{2} \mathrm{O}$. The decreasing abundance towards high temperatures is due to the increasingly efficient reformation of $\mathrm{H}_{2} \mathrm{O}$ after destruction due to cosmic rays. 


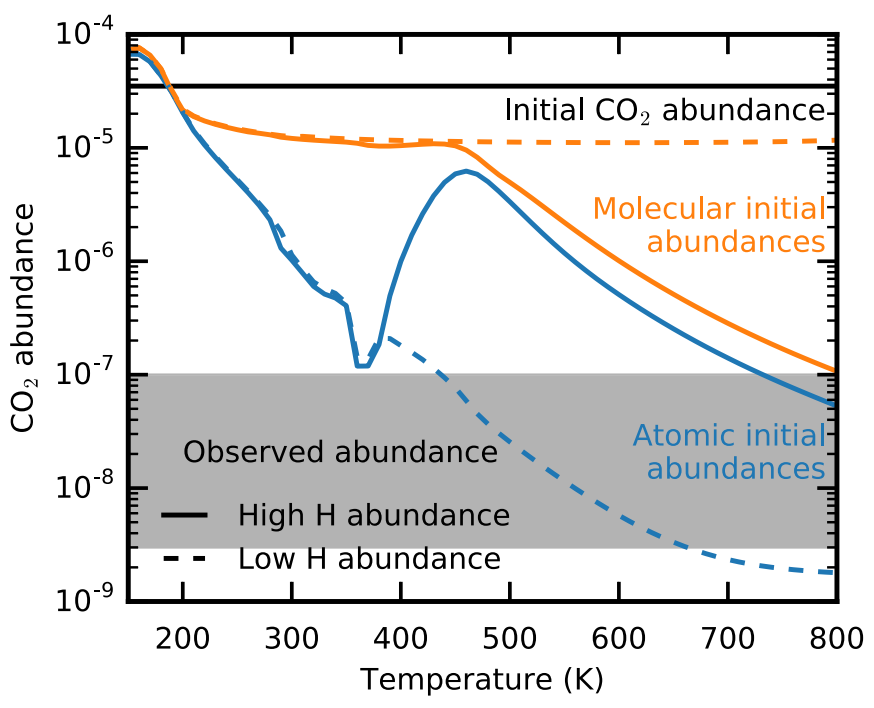

Fig. B.1. $\mathrm{CO}_{2}$ abundance from a gas-phase chemical network as function of temperature after $1 \mathrm{Myr}$ of evolution. The density is $10^{12} \mathrm{~cm}^{-3}$.

\section{B.2. Grain surface chemistry between the $\mathrm{H}_{2} \mathrm{O}$ and $\mathrm{CO}_{2}$ icelines}

Section B.1 investigates the gas-phase chemistry of $\mathrm{CO}_{2}$ in the region within the $\mathrm{H}_{2} \mathrm{O}$ iceline, that is, above $150 \mathrm{~K}$. In this region all species will be primarily in the gas-phase and grain surface chemistry is thus expected to be less important. Between the $\mathrm{CO}_{2}$ and $\mathrm{H}_{2} \mathrm{O}$ icelines The efficiency of the destruction of gaseous $\mathrm{CO}_{2}$ is estimated by investigating the chemical pathways in a full chemical model. The network from Walsh et al. (2014) and Eistrup et al. (2016) was used. A point model representative for the conditions between the $\mathrm{H}_{2} \mathrm{O}$ and $\mathrm{CO}_{2}$ iceline was run. The number density used is $10^{13} \mathrm{~cm}^{-3}$, the temperature $100 \mathrm{~K}$ and the cosmic ray ionisation rate $10^{-17} \mathrm{~s}^{-1}$. There was no external UV in our model. After running the model for $1 \mathrm{Myr}$ to get equilibrium abundances of atomic and ionised species, the chemical pathways of oxygen carrying species are examined.

For each oxygen carrying molecule that could be created from a destruction of $\mathrm{CO}_{2}$, we define the destruction efficiency
Table B.2. Chemical pathways from molecules possibly created by the destruction of $\mathrm{CO}_{2}$.

\begin{tabular}{lll}
\hline \hline $\begin{array}{l}\text { First generation } \\
\text { molecule }\end{array}$ & $\begin{array}{l}\text { Destruction } \\
\text { efficiency }\end{array}$ & $\begin{array}{l}\text { Stable resulting } \\
\text { molecules }\end{array}$ \\
\hline $\mathrm{CO}_{2}^{+}, \mathrm{HCO}_{2}^{+}$ & $0 \%$ & $\mathrm{CO}_{2}$ \\
$\mathrm{OH}$ & $27 \%$ & $\mathrm{CO}_{2}, \mathrm{O}_{2}, \mathrm{H}_{2} \mathrm{O}_{2}$ \\
$\mathrm{O}^{+}, \mathrm{OH}^{+}, \mathrm{H}_{2} \mathrm{O}^{+}, \mathrm{H}_{3} \mathrm{O}^{+}$ & $100 \%$ & $\mathrm{H}_{2} \mathrm{O}$ \\
$\mathrm{O}, \mathrm{NO}, \mathrm{HNO}$ & $82 \%$ & $\mathrm{O}_{2}, \mathrm{CO}_{2}, \mathrm{H}_{2} \mathrm{O}_{2}$ \\
$\mathrm{SO}, \mathrm{SO}_{2}$ & $100 \%$ & $\mathrm{O}_{2}$ \\
$\mathrm{OCN}$ & $0 \%$ & $\mathrm{CO}_{2}$ \\
$\mathrm{HCNO}$ & $100 \%$ & $\mathrm{O}_{2}$ \\
$\mathrm{SiO}$ & $100 \%$ & $\mathrm{SiO}$ \\
\hline
\end{tabular}

as the fraction the oxygen atoms that is incorporated into a stable molecule, for which we take that a cosmic ray induced process is needed to destroy the molecule. Here, pathways leading to incorporation of oxygen into $\mathrm{CO}$ are neglected, as these pathways are dominated by the availability of atomic $\mathrm{C}$, which must originate from an other $\mathrm{CO}$ molecule. Pathways towards $\mathrm{CO}$ are generally very minor. Destruction efficiencies are tabulated in Table B.2.

From the efficiencies it can be seen that direct ionisation or protonation of $\mathrm{CO}_{2}$ is not an effective way to destroy it since $\mathrm{CO}_{2}$ will be reformed from the products in most cases. Only if an oxygen atom can be removed from the central carbon, is there a chance that the oxygen will be locked up into something other than $\mathrm{CO}_{2}$. In these cases the efficiency is $27 \%$ (for $\mathrm{OH}$ ) or higher.

\section{Appendix C: Viscous evolution and grain growth}

Figures C.1 through C.9 show model results for the model with grain growth and radial drift, but without any destruction rate. $\alpha$ values of $10^{-2}, 10^{-3}$ and $10^{-4}$ are shown, fragmentation velocities of 1,10 and $100 \mathrm{~m} \mathrm{~s}^{-1}$ are used. The features seen in ice abundance for the higher fragmentation velocity models is due to a numerical instability in the grain growth algorithm, this does not influence our results. 

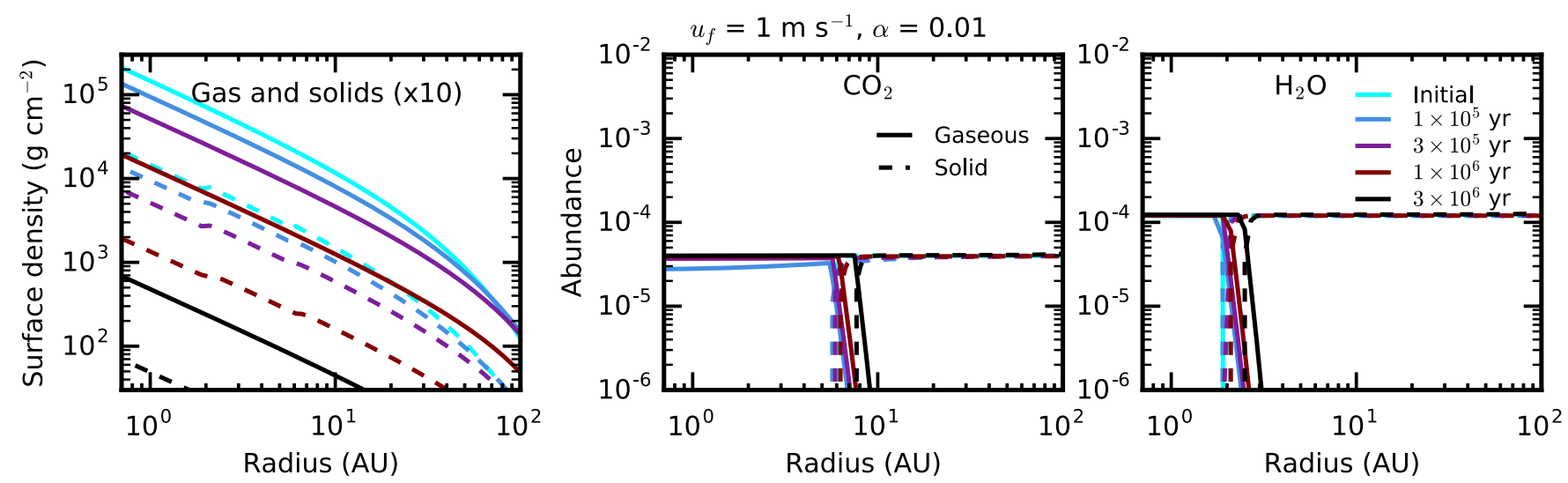

Fig. C.1. Time evolution series for a model with grain growth. This model assumes an $\alpha$ of $10^{-2}$. The fragmentation velocity for this model is $1 \mathrm{~m} \mathrm{~s}^{-1}$. Panels as in Fig. 3.
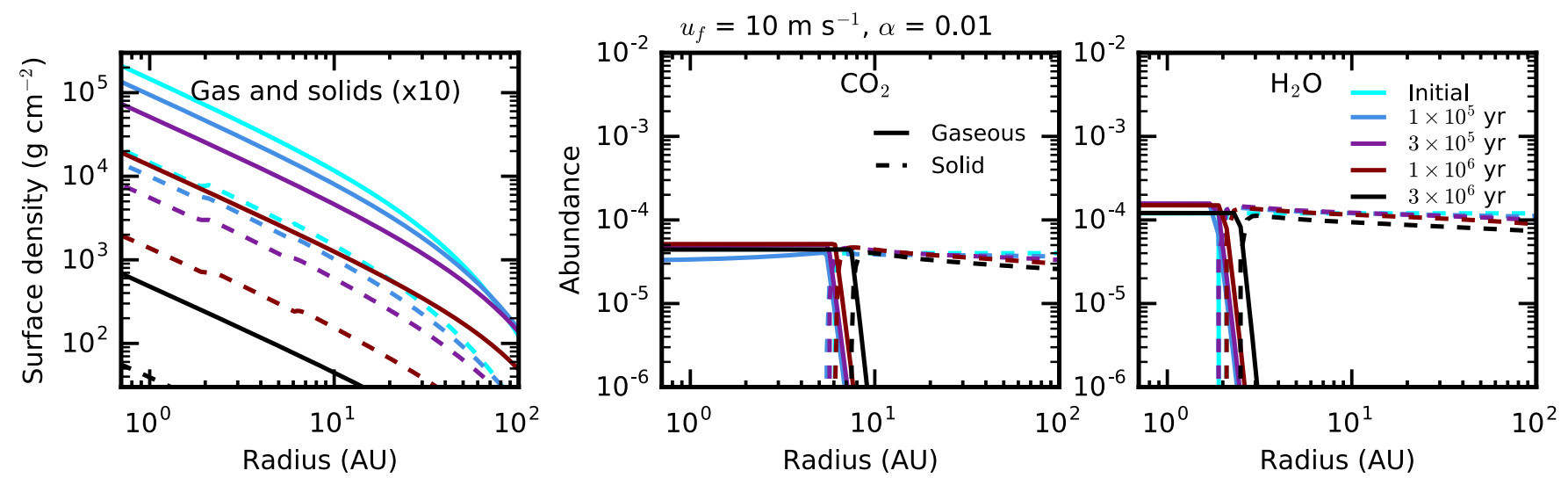

Fig. C.2. Time evolution series for a model with grain growth. This model assumes an $\alpha$ of $10^{-2}$. The fragmentation velocity for this model is $1 \mathrm{~m} \mathrm{~s}^{-1}$. Panels as in Fig. 3.
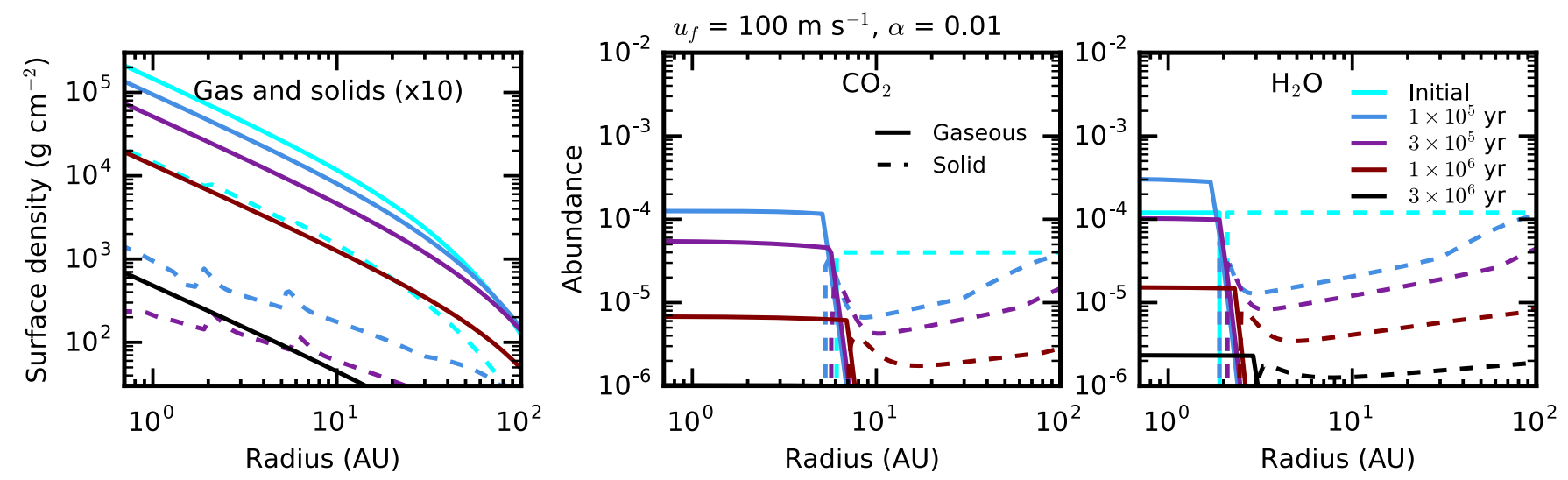

Fig. C.3. Time evolution series for a model with grain growth. This model assumes an $\alpha$ of $10^{-2}$. The fragmentation velocity for this model is $1 \mathrm{~m} \mathrm{~s}^{-1}$. Panels as in Fig. 3. 
A. D. Bosman et al.: Efficiency of radial transport of ices: the case of $\mathrm{CO}_{2}$
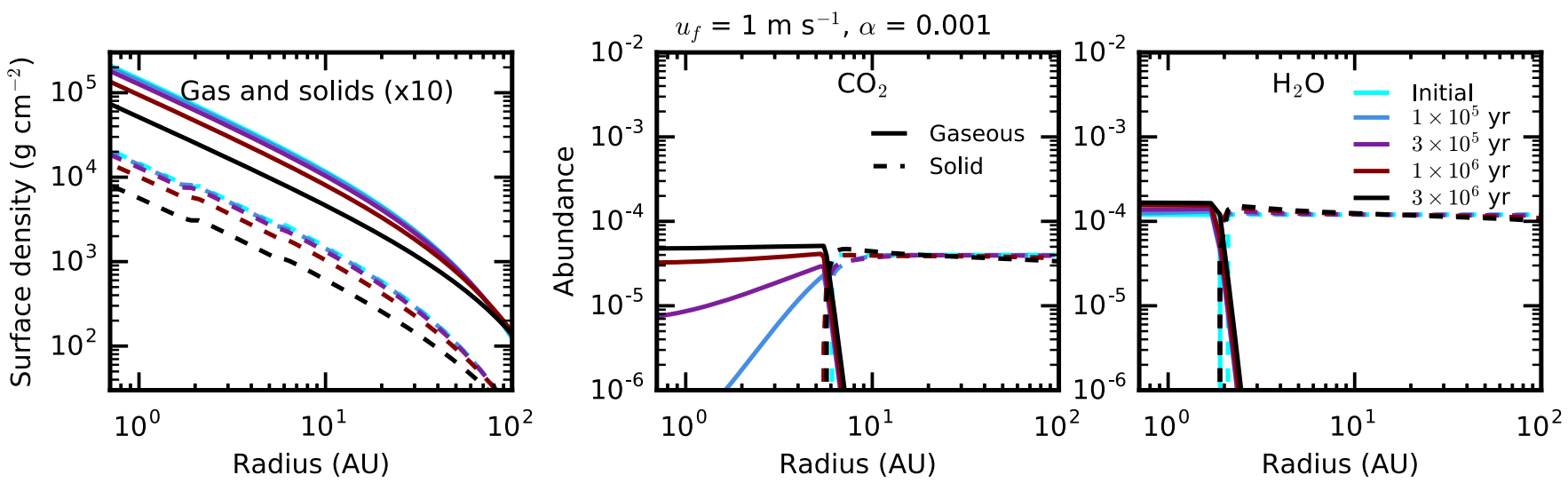

Fig. C.4. Time evolution series for a model with grain growth. This model assumes an $\alpha$ of $10^{-3}$. The fragmentation velocity for this model is $1 \mathrm{~m} \mathrm{~s}^{-1}$. Panels as in Fig. 3.
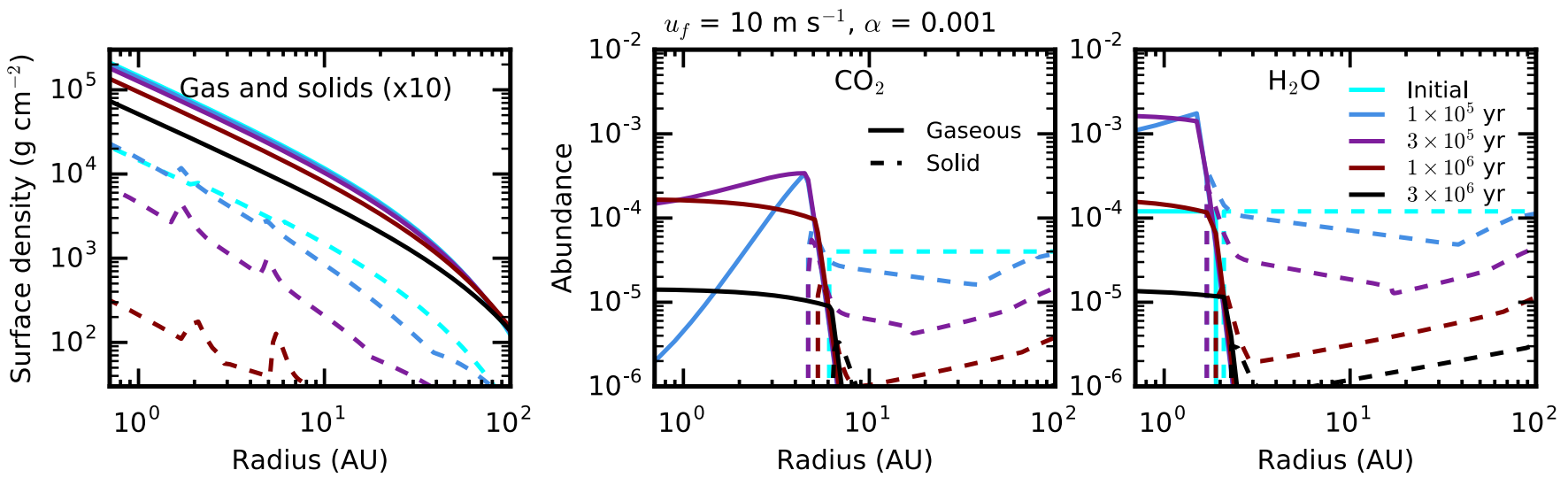

Fig. C.5. Time evolution series for a model with grain growth. This model assumes an $\alpha$ of $10^{-3}$. The fragmentation velocity for this model is $10 \mathrm{~m} \mathrm{~s}^{-1}$. Panels as in Fig. 3.
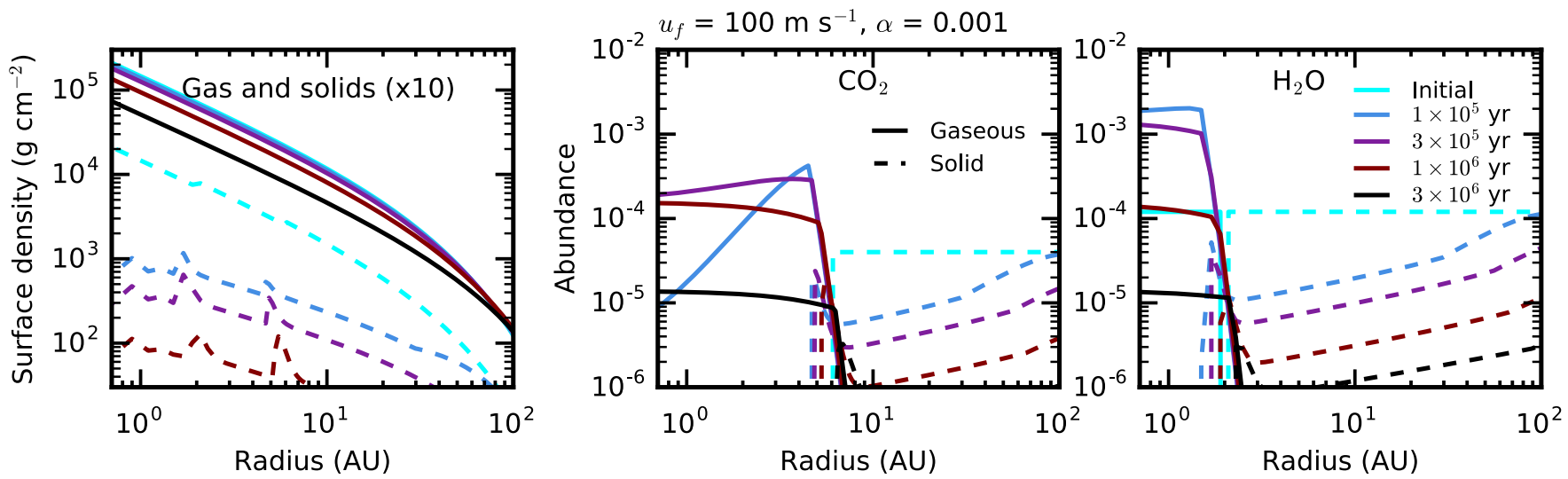

Fig. C.6. Time evolution series for a model with grain growth. This model assumes an $\alpha$ of $10^{-3}$. The fragmentation velocity for this model is $100 \mathrm{~m} \mathrm{~s}^{-1}$. Panels as in Fig. 3. 

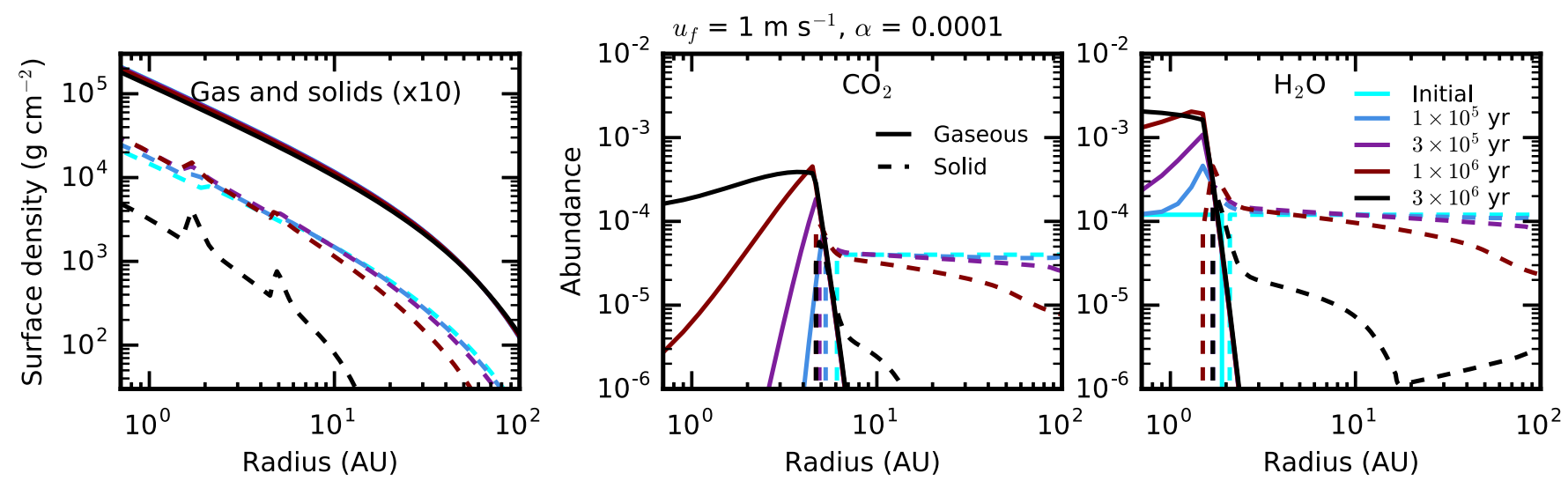

Fig. C.7. Time evolution series for a model with grain growth. This model assumes an $\alpha$ of $10^{-4}$. The fragmentation velocity for this model is $1 \mathrm{~m} \mathrm{~s}^{-1}$. Panels as in Fig. 3.
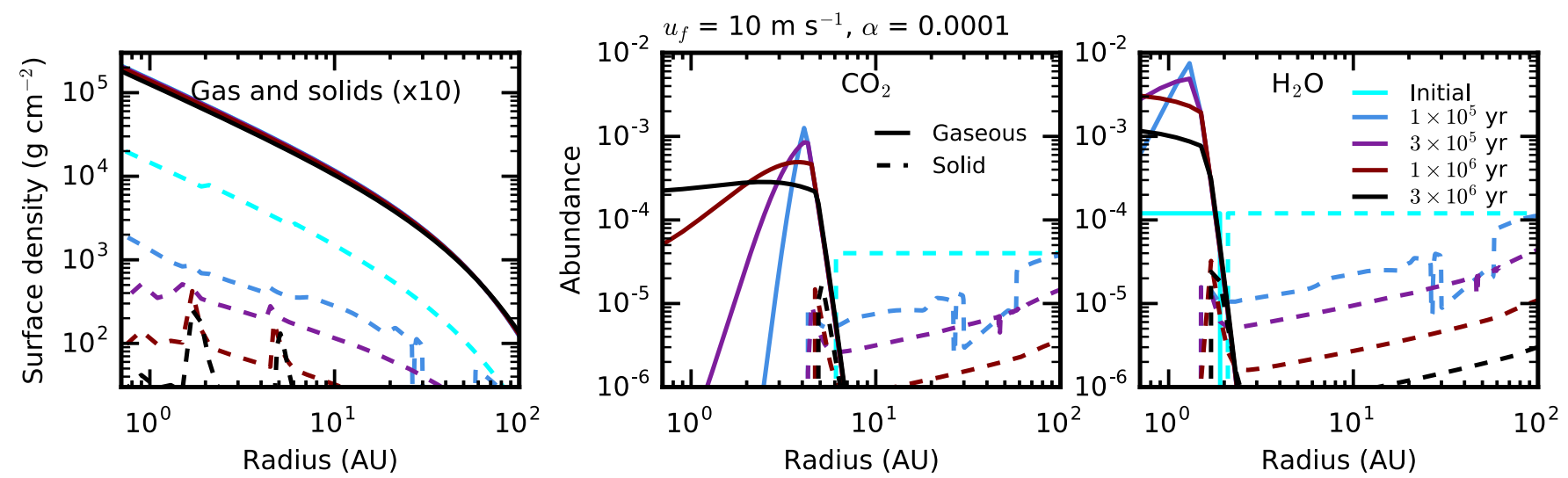

Fig. C.8. Time evolution series for a model with grain growth. This model assumes an $\alpha$ of $10^{-4}$. The fragmentation velocity for this model is $10 \mathrm{~m} \mathrm{~s}^{-1}$. Panels as in Fig. 3.
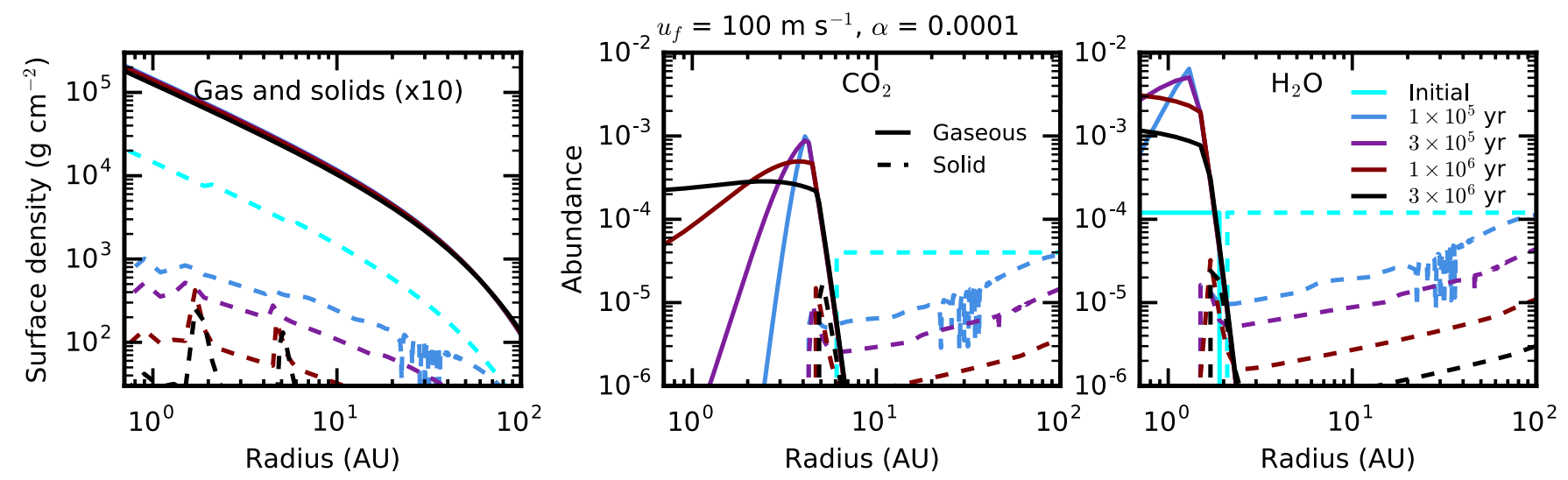

Fig. C.9. Time evolution series for a model with grain growth. This model assumes an $\alpha$ of $10^{-4}$. The fragmentation velocity for this model is $100 \mathrm{~m} \mathrm{~s}^{-1}$. Panels as in Fig. 3. 